\title{
ON A MODEL SYSTEM FOR THE OBLIQUE INTERACTION OF INTERNAL GRAVITY WAVES *
}

\author{
Jean-Claude Saut ${ }^{1}$ and Nikolay Tzvetkov ${ }^{1}$
}

\begin{abstract}
We give local and global well-posedness results for a system of two Kadomtsev-Petviashvili (KP) equations derived by R. Grimshaw and Y. Zhu to model the oblique interaction of weakly nonlinear, two dimensional, long internal waves in shallow fluids. We also prove a smoothing effect for the amplitudes of the interacting waves. We use the Fourier transform restriction norms introduced by J. Bourgain and the Strichartz estimates for the linear KP group. Finally we extend the result of [3] for lower order perturbation of the system in the absence of transverse effects.
\end{abstract}

Mathematics Subject Classification. 35Q07, 35Q53, 76B15.

Received: October 14, 1999.

\section{INTRODUCTION}

This paper is directly motivated by the work of Grimshaw and Zhu [15], where a model is presented for the oblique strong interaction of weakly, two dimensional, nonlinear, long internal gravity waves in both shallow and deep fluids. The model consists of two coupled Kadomtsev-Petviashvili (KP) equations (shallow fluid) or of two coupled "intermediate long wave" equations (ILW) (deep fluid). The interaction of internal gravity waves propagating in one horizontal direction has been considered by Gear and Grimshaw [12]. Rigorous mathematical results in this case have been obtained by Bona et al. [4], Ash, Cohen and Wang [3], Albert, Bona and Saut [1] (cf. also Appendix A of the present paper for a complement to the paper [3]).

More precisely we are interested here in the case which Grimshaw and Zhu refer as the "strong interaction I" type, in the shallow water situation ( $c f$. [15] Sect. 3.2). Thus we require that the 2 interacting modes $n$ and $m$ have closed phase speeds, $c_{n} \sim c_{m}$, and that the angle $\delta$ between the waves (which propagate in the $x-y$ plane) is closed to zero. In this case, Grimshaw and Zhu using a multi-scale perturbation method, found that the vertical displacement $\xi$ of the fluid is given by

$$
\xi=\alpha\left\{A_{n}\left(\tau, \sigma_{1}, \sigma_{2}, \theta\right) \phi_{n}(z)+A_{m}\left(\tau, \sigma_{1}, \sigma_{2}, \theta\right) \phi_{m}(z)\right\}+\alpha^{2} \xi_{1}+O\left(\alpha^{3}\right) .
$$

Here the small parameter $\alpha$ is a measure of the wave amplitude, $\theta=X-c_{n} T$ is the phase variable, $\tau=\alpha T$, $\sigma_{1}=\alpha X, \sigma_{2}=\alpha^{\frac{1}{2}} X$ are respectively the long time and space variables. They are related to the physical

\footnotetext{
Keywords and phrases. Internal gravity waves, Kadomtsev-Petviashvili equations, Cauchy problem.

* Dedicated to Roger Temam for his 60th birthday

1 Analyse numérique et EDP, Université de Paris-Sud, Bât. 425, 91405 Orsay Cedex, France.

e-mail: Jean-Claude.saut@math.u-psud.fr, Nikolay.tzvetkov@math.u-psud.fr
} 
variables $(x, y, t)$ by

$$
X=\varepsilon x, \quad Y=\varepsilon y, \quad T=\varepsilon t,
$$

where $\varepsilon$ is a small parameter representing the dispersive effects, namely the ratio of vertical to horizontal scales. A KdV balance is assumed between dispersion and nonlinearity, i.e. $\alpha=\varepsilon^{2}$. Moreover $c_{m}$ is close to $c_{n}$, namely $c_{m}=c_{n}(1+\alpha V)$, where $V$ is $O(1)$ with respect to $\alpha$. In (1) $\phi_{j}, j=m, n$ is the $j^{t h}$ mode of the vertical mode function $\phi=\phi(z)$ with corresponding phase speed $c_{j}$ (they are eigenfunctions of some linear eigenvalue problem which we will not specify here). Finally, the amplitudes $A_{m}$ and $A_{n}$ are shown in [15] to satisfy the system of evolution equations

$$
\left\{\begin{array}{c}
\left.\frac{I_{n}}{c_{n}^{2}}\left\{2 \frac{\partial}{\partial \theta}\left(\frac{1}{c_{n}} \frac{\partial A_{n}}{\partial \tau}+\frac{\partial A_{n}}{\partial \sigma_{1}}+\lambda_{n} \frac{\partial^{3} A_{n}}{\partial \theta^{3}}+\nu_{n} A_{n} \frac{\partial A_{n}}{\partial \theta}\right)+\frac{\partial^{2} A_{n}}{\partial \sigma_{2}^{2}}\right)\right\} \\
+3 \mu_{n n m} \frac{\partial^{2}}{\partial \theta^{2}}\left(A_{n} A_{m}\right)+3 \mu_{n m m} \frac{\partial}{\partial \theta}\left(A_{m} \frac{\partial A_{m}}{\partial \theta}\right)+\lambda_{n m} \frac{\partial^{4} A_{m}}{\partial \theta^{4}}=0 \\
\left.\frac{I_{m}}{c_{m}^{2}}\left\{2 \frac{\partial}{\partial \theta}\left(\frac{1}{c_{n}} \frac{\partial A_{m}}{\partial \tau}+V \frac{\partial A_{m}}{\partial \theta}+\frac{\partial A_{m}}{\partial \sigma_{1}}+\lambda_{m} \frac{\partial^{3} A_{m}}{\partial \theta^{3}}+\nu_{m} A_{m} \frac{\partial A_{m}}{\partial \theta}\right)+\frac{\partial^{2} A_{m}}{\partial \sigma_{2}^{2}}\right)\right\} \\
+3 \mu_{n m m} \frac{\partial^{2}}{\partial \theta^{2}}\left(A_{n} A_{m}\right)+3 \mu_{n n m} \frac{\partial}{\partial \theta}\left(A_{n} \frac{\partial A_{n}}{\partial \theta}\right)+\lambda_{m n} \frac{\partial^{4} A_{n}}{\partial \theta^{4}}=0
\end{array}\right.
$$

where

$$
\begin{gathered}
\mu_{s k l}=\int_{-h}^{0} \rho_{0} \frac{\partial \phi_{s}}{\partial z} \frac{\partial \phi_{k}}{\partial z} \frac{\partial \phi_{l}}{\partial z} \mathrm{~d} z, \quad \lambda_{k l}=\int_{-h}^{0} \rho_{0} \phi_{k} \phi_{l}, \quad I_{k} \nu_{k}=\frac{3}{2} c_{k}^{2} \int_{-h}^{0} \rho_{0}\left(\frac{\partial \phi_{k}}{\partial z}\right)^{3} \mathrm{~d} z, \\
I_{k} \lambda_{k}=\frac{1}{2} c_{k}^{2} \int_{-h}^{0} \rho_{0} \phi_{k}^{2}, \quad \delta_{k l} I_{k}=c_{k}^{2} \int_{-h}^{0} \rho_{0} \frac{\partial \phi_{k}}{\partial z} \frac{\partial \phi_{l}}{\partial z} \mathrm{~d} z .
\end{gathered}
$$

Here $k, l, s \in\{m, n\}$ and $\delta_{k l}$ is the Kronecker symbol. First we observe as in [15] that by transforming to a reference frame traveling with speed $c_{n}$ we may ignore the dependence on $\sigma_{1}$ in the system (2) up to higher order terms.

Then the system (2) can be rewritten in the form

$$
\left\{\begin{array}{c}
\left(u_{t}+a_{11} u_{x x x}+a_{12} v_{x x x}+b_{1}(u v)_{x}+b_{2} u u_{x}+b_{3} v v_{x}\right)_{x}+u_{y y}=0 \\
\left(v_{t}+a_{21} u_{x x x}+a_{22} v_{x x x}+r v_{x}+b_{4}(u v)_{x}+b_{5} u u_{x}+b_{6} v v_{x}\right)_{x}+v_{y y}=0 \\
u(0, x, y)=u_{0}(x, y), \quad v(0, x, y)=v_{0}(x, y)
\end{array}\right.
$$

where

$$
\begin{gathered}
t=\tau, \quad x=\theta, \quad y=\sigma_{2}, \\
u(t, x, y)=A_{n}\left(\tau, \theta,\left(2 / c_{n}\right)^{\frac{1}{2}} \sigma_{2}\right), \quad v(t, x, y)=A_{m}\left(\tau, \theta,\left(2 / c_{m}\right)^{\frac{1}{2}} \sigma_{2}\right), \\
a_{11}=\lambda_{n} c_{n}, \quad a_{12}=\frac{\lambda_{n m} c_{n}^{3}}{2 I_{n}}, \quad a_{21}=\frac{\lambda_{m n} c_{m}^{2} c_{n}}{2 I_{m}}, \quad a_{22}=\lambda_{m} c_{n}, \\
b_{1}=\frac{3 \mu_{n n m} c_{n}^{3}}{2 I_{n}}, \quad b_{2}=c_{n} \nu_{n}, \quad b_{3}=\frac{3 \mu_{n m m} c_{n}^{3}}{2 I_{n}} \\
b_{4}=\frac{3 \mu_{n m m} c_{m}^{2} c_{n}}{2 I_{m}}, \quad b_{5}=\frac{3 \mu_{n n m} c_{m}^{2} c_{n}}{2 I_{m}}, \quad b_{6}=c_{n} \nu_{m}
\end{gathered}
$$




$$
r=c_{n} V=\left(c_{m}-c_{n}\right) / \alpha .
$$

Assuming that $c_{m}-c_{n}$ is very small comparatively to $\alpha$ (cf. [4]) we will take $r=0$ in (3) from now on.

We are interested in the local well-posedness of the Cauchy problem (3) in Sobolev type spaces based on $L^{2}$. In general local well-posedness in the classical Sobolev spaces $H^{s}$ for $s$ small enough may allow us to establish global existence using the conservation laws of the system. It is easy to see that the $L^{2}$ norms of the solutions of (2) are conserved. Another conservation law of (2) is due to the time translation invariance of the system, i.e. the energy. The quadratic part of the energy of (2) is not positive definite in the relevant values of the parameters, and therefore the only reasonable conservation law which may allow to establish global existence seems to be the conservation of the $L^{2}$ norm. In $[6,7]$ Bourgain developed a new method for studying the local well-posedness of some nonlinear evolution equations. One of the main points of the method is the introduction of Fourier transform restriction norms. The method was also applied for KP-II equation $(c f .[8,18,24,25])$. In [8] local well-posedness for KP-II in $L^{2}$ is shown. The proof uses a suitable dyadic decomposition associated to the symbol of the linearized operator. The arguments are mainly performed for the case of periodic boundary conditions. But the proof could be adapted for data in $\mathbb{R}^{2}$. In the present paper we are going to show that (2) is globally well-posed for data in $L^{2}$. In the nonlinear estimates, similarly to [25], we shall make use of the dispersive properties of (2) (Strichartz type inequalities) and of some simple calculus inequalities similarly to [20] for the KdV equation, [21] for the nonlinear Schrödinger equation or [18] for the KP-II equation. As in $[18,25]$, the proof turns out to be simpler that the one of [8]. We also shall prove a smoothing effect in the $x$ variable for (2). Actually we shall show that the linear evolution of the system propagates the main singularity. More precisely in the main direction of propagation the solution turns out to be a compact perturbation of the linear evolution. The main goal of this paper is to prove the following Theorem.

Theorem 1. Suppose that $a_{11} a_{22}-a_{12} a_{21}>0$. Then for any

$$
\left(u_{0}, v_{0}\right) \in L^{2}\left(\mathbb{R}^{2}\right) \times L^{2}\left(\mathbb{R}^{2}\right),
$$

such that

$$
|\xi|^{-1} \mathcal{F}\left(u_{0}\right)(\xi, \eta) \in \mathcal{S}^{\prime}, \quad|\xi|^{-1} \mathcal{F}\left(v_{0}\right)(\xi, \eta) \in \mathcal{S}^{\prime}
$$

there exists a unique solution $(u, v)$ of the Cauchy problem (3). In addition the solution satisfy

$$
(u, v) \in C\left(\mathbb{R} ; L^{2}\left(\mathbb{R}^{2}\right)\right) \times C\left(\mathbb{R} ; L^{2}\left(\mathbb{R}^{2}\right)\right) .
$$

Moreover the following decomposition of $(u, v)$ at any time $t$ holds

$$
(u(t), v(t))=\left(u^{1}(t), v^{1}(t)\right)+\left(u^{2}(t), v^{2}(t)\right)
$$

where $\left(u^{1}(t), v^{1}(t)\right) \in L^{2}\left(\mathbb{R}^{2}\right) \times L^{2}\left(\mathbb{R}^{2}\right)$ is the free evolution of the system and

$$
\left(u^{2}(t), v^{2}(t)\right) \in H^{s, 0}\left(\mathbb{R}^{2}\right) \times H^{s, 0}\left(\mathbb{R}^{2}\right) \text { for } s \in\left[0, \frac{1}{29}\right) .
$$

Remark 1. Coming back to the notations of (2), the condition $a_{11} a_{22}-a_{12} a_{21}>0$ is equivalent to $c_{n}^{2} c_{m}^{2} \lambda_{m n}^{2}<$ $4 I_{n} I_{m} \lambda_{n} \lambda_{m}$.

The paper is organized as follows. In Section 2, we first diagonalize the linear part of (2). Then using the parabolic regularisation method similarly to [17] we prove the local well-posedness of (2) in anisotropic Sobolev spaces $\widetilde{H}^{s_{1}, s_{2}}\left(\mathbb{R}^{2}\right)$ with regularity $s_{1}>3 / 2, s_{2}>1 / 2$. This is an improvement of the result of [17] which states local well-posedness for KP equations in the classical Sobolev spaces $H^{s}\left(\mathbb{R}^{2}\right), s>2$. We must set the restriction on the pair $\left(s_{1}, s_{2}\right)$ in order to control the $L^{\infty}$-norm of the $x$ derivative of the solution. On the other hand, the result does not depend on the nature (KP-I or KP-II) of the system. Sections 3 and 4 are devoted to the case 
when the system (2) consists of two coupled KP-II equations. In Section 3.1 we first define the functional spaces where the solutions are expected to belong. Then we state the linear and nonlinear estimates. Section 3.2 is devoted to the proof of the nonlinear estimate. First we state the Strichartz inequalities injected into the framework of Bourgain's spaces and some simple calculus inequalities needed for the proof. The rest of the Section contains the proof of the integral representation of the nonlinear estimate. In Section 3.3 we apply a fixed point argument to prove Theorem 3.1. Section 3.4 is devoted to the proof of the smoothing effect. In Section 4 we extend the global well-posedness to spaces of higher smoothness. In Appendix A we consider the system of Gear-Grimshaw ( $c f .[12])$, i.e. two coupled KdV equations. We treat the case when the difference of the speeds of the two interacting modes is not necessary small comparatively to a measure of the wave amplitude. Finally in Appendix B we prove the estimate needed for the local well-posedness in anisotropic Sobolev spaces of Section 2.

We shall use the following notations. By ${ }^{\wedge}$ or $\mathcal{F}$ we denote the Fourier transform, while by $\mathcal{F}^{-1}$ the inverse transform. $\|\cdot\|_{L^{p}}$ denotes the norm in the Lebesgue space $L^{p}$, while $H^{s_{1}, s_{2}}\left(\mathbb{R}^{2}\right)$ denotes the anisotropic Sobolev space equipped with the norm

$$
\|u\|_{H^{s_{1}, s_{2}}}=\left\|\langle\xi\rangle^{s_{1}}\langle\eta\rangle^{s_{2}} \widehat{u}(\xi, \eta)\right\|_{L_{(\xi, \eta)}^{2}},
$$

where $\langle\rangle=.\left(1+|.|^{2}\right)^{\frac{1}{2}}$. With $H^{s}$ we denote the classical Sobolev spaces. The notation $a \pm 0$ means $a \pm \varepsilon$ for arbitrary small $\varepsilon>0$. The operators $D_{x}$ and $D_{y}$ are defined respectively by $D_{x}=\frac{1}{i} \partial_{x}, D_{y}=\frac{1}{i} \partial_{y}$. Constants are denoted by $c$ and may change from line to line.

We thank R. Grimshaw for helpful explanations on the system (2) and I. Gallagher for useful discussions on the anisotropic Littlewood-Paley decompositions.

\section{A LOCAL EXISTENCE THEOREM}

Set

$$
\begin{gathered}
g=(u, v)^{t}, \quad A=\left(\begin{array}{cc}
a_{11} & a_{12} \\
a_{21} & a_{22}
\end{array}\right), \\
f(u, v)=\left(b_{1}(u v)_{x}+b_{2} u u_{x}+b_{3} v v_{x}, b_{4}(u v)_{x}+b_{5} u u_{x}+b_{6} v v_{x}\right)^{t} .
\end{gathered}
$$

Therefore the system (3) can be written as$$
\left(\partial_{t} \partial_{x}+\partial_{y}^{2}\right) g+A \partial_{x}^{4} g+\partial_{x} f(u, v)=0 .
$$

Note that

$$
a_{12} a_{21}=\frac{\lambda_{m n}^{2} c_{m}^{3} c_{n}^{3}}{2 I_{m} I_{n}}>0 .
$$

There exists $T \in \mathrm{GL}(2)$ such that $T^{-1} A T=\operatorname{diag}\left(\gamma^{+}, \gamma^{-}\right)$, where $\gamma^{ \pm}$are the eigenvalues of $A$

$$
\gamma^{ \pm}=\frac{1}{2}\left(a_{11}+a_{22} \pm \sqrt{\left(a_{11}-a_{22}\right)^{2}+4 a_{12} a_{21}}\right) .
$$

We have that $\gamma^{+}>0$ and the sign of $\gamma^{-}$is that of $a_{11} a_{22}-a_{12} a_{21}$. Now setting $g=T h$, where $h=\left(w^{+}, w^{-}\right)$ we arrive at the system

$$
\left(\partial_{t} \partial_{x}+\partial_{y}^{2}\right) h+\operatorname{diag}\left(\gamma^{+}, \gamma^{-}\right) \partial_{x}^{4} h+\partial_{x} F\left(w^{+}, w^{-}\right)=0,
$$

where

$$
\begin{aligned}
F\left(w^{+}, w^{-}\right) & =\left(F^{+}\left(w^{+}, w^{-}\right), F^{-}\left(w^{+}, w^{-}\right)\right)^{t} \\
& =\left(\bar{b}_{1}\left(w^{+} w^{-}\right)_{x}+\bar{b}_{2} w^{+} w_{x}^{+}+\bar{b}_{3} w^{-} w_{x}^{-}, \bar{b}_{4}\left(w^{+} w^{-}\right)_{x}+\bar{b}_{5} w^{+} w_{x}^{+}+\bar{b}_{6} w^{-} w_{x}^{-}\right)^{t}
\end{aligned}
$$


with some constants $\bar{b}_{1}, \ldots, \bar{b}_{6}$. Note that (5) has a structure of 2 coupled KP-II equations $\left(\gamma^{-}>0\right)$ or of a KP-II equation coupled with a KP-I equation $\left(\gamma^{-}<0\right)$.

Since the symbol of the linearized operator has a singularity near the origin we introduce an anisotropic Sobolev space $\widetilde{H}^{s_{1}, s_{2}}\left(\mathbb{R}^{2}\right)$ spaces similarly to [22], equipped with the norm

$$
\|u\|_{\tilde{H}^{s_{1}, s_{2}}}^{2}=\int_{\mathbb{R}^{2}}\left(1+|\xi|^{-1}\right)^{2}\langle\xi\rangle^{2 s_{1}}\langle\eta\rangle^{2 s_{2}}|\widehat{u}(\xi, \eta)|^{2} \mathrm{~d} \xi \mathrm{d} \eta .
$$

Note that (formally) any $u \in \tilde{H}^{s_{1}, s_{2}}\left(\mathbb{R}^{2}\right)$ has zero $x$ mean value. Now we write (5) in the form

$$
\left(h_{t}+\operatorname{diag}\left(\gamma^{+}, \gamma^{-}\right) h_{x x x}+A(h) h_{x}\right)_{x}+h_{y y}=0,
$$

where the matrix $A$ is defined by

$$
A(h)=\left(\begin{array}{cc}
\bar{b}_{2} w^{+}+\bar{b}_{1} w^{-} & \bar{b}_{1} w^{+}+\bar{b}_{3} w^{-} \\
\bar{b}_{5} w^{+}+\bar{b}_{4} w^{-} & \bar{b}_{4} w^{+}+\bar{b}_{6} w^{-}
\end{array}\right) .
$$

Note that in $(6), A(0)$ is the zero matrix and that $A(h)$ is smooth. These are the conditions which allow us to apply the parabolic regularization method to the system (6), which is a vectorial variant of the equation studied in [17]. Consider a regularized version of (6)

$$
\left(h_{t}-\varepsilon \Delta h+\operatorname{diag}\left(\gamma^{+}, \gamma^{-}\right) h_{x x x}+A(h) h_{x}\right)_{x}+h_{y y}=0
$$

where $\Delta$ is the Laplace operator on $\mathbb{R}^{2}$ and $\varepsilon$ is a small positive parameter. Due to the parabolic character of (7) and using Proposition 2.1 below, for any $s_{1}>3 / 2$ and $s_{2}>1 / 2$ we have a unique solution $h^{\varepsilon} \in$ $C\left([0, T] ; \tilde{H}^{s_{1}, s_{2}}\left(\mathbb{R}^{2}\right)\right)$ of $(7)$ with initial data $h^{0}=h(0)$. Here $T$ depends on $\varepsilon$ and $\left\|h^{0}\right\|_{\tilde{H}^{s_{1}, s_{2}}}$. We have to show that $T$ can be chosen independent of $\varepsilon$. Multiplying (7) by $h$ and integration over $(x, y)$ we obtain

$$
\frac{\mathrm{d}}{\mathrm{d} t}\left\|h^{\varepsilon}(t)\right\|_{H^{s_{1}, s_{2}}}^{2}=-2 \varepsilon\left\|\nabla h^{\varepsilon}\right\|_{H^{s_{1}, s_{2}}}^{2}+2\left(h^{\varepsilon}, A\left(h^{\varepsilon}\right) h_{x}^{\varepsilon}\right)_{s_{1}, s_{2}}
$$

where $(.,)_{s_{1}, s_{2}}$ stays for the $H^{s_{1}, s_{2}}$ scalar product. Now we state a Proposition which will be proved in Appendix B.

Proposition 2.1. Let $s_{1}>1 / 2$ and $s_{2}>1 / 2$. Then the space $H^{s_{1}, s_{2}}\left(\mathbb{R}^{2}\right)$ is an algebra.

Using Proposition 2.1 we obtain for $s_{1}>3 / 2$ and $s_{2}>1 / 2$

$$
\frac{\mathrm{d}}{\mathrm{d} t}\left\|h^{\varepsilon}(t)\right\|_{H^{s_{1}, s_{2}}}^{2} \leq c\left\|h^{\varepsilon}(t)\right\|_{H^{s_{1}, s_{2}}}^{3}
$$

Similarly we obtain

$$
\frac{\mathrm{d}}{\mathrm{d} t}\left\|\partial_{x}^{-1} h^{\epsilon}(t)\right\|_{H^{s_{1}, s_{2}}}^{2} \leq c\left\|\partial_{x}^{-1} h^{\epsilon}(t)\right\|_{H^{s_{1}, s_{2}}}^{3}
$$

Hence

$$
\left\|h^{\epsilon}(t)\right\|_{\tilde{H}^{s_{1}, s_{2}}}^{2} \leq\left\|h^{\epsilon}(0)\right\|_{\tilde{H}^{s_{1}, s_{2}}}^{2}+c \int_{0}^{t}\left\|h^{\epsilon}(\tau)\right\|_{\tilde{H}^{s_{1}, s_{2}}}^{3} \mathrm{~d} \tau .
$$

Furthermore

$$
\left\|h^{\epsilon}(t)\right\|_{\tilde{H}^{s_{1}, s_{2}}} \leq \frac{c\left\|h^{0}\right\|_{\tilde{H}^{s_{1}, s_{2}}}}{c-t\left\|h^{0}\right\|_{\tilde{H}^{s_{1}, s_{2}}}} .
$$


Once we obtain (8) it remains to use standard arguments (cf. [17]) to derive local well-posedness of (6) in $\tilde{H}^{s_{1}, s_{2}}, s_{1}>3 / 2, s_{2}>1 / 2$. To the solutions of (6) will correspond solutions of the original system (2). Hence we can obtain the next Theorem.

Theorem 2.1. Let $s_{1}>3 / 2, s_{2}>1 / 2$ and

$$
(u(0), v(0)) \in \tilde{H}^{s_{1}, s_{2}}\left(\mathbb{R}^{2}\right) \times \tilde{H}^{s_{1}, s_{2}}\left(\mathbb{R}^{2}\right) .
$$

Then there exist $T>0$ and a unique solution $(u, v)$ of the Cauchy problem (3). In addition

$$
(u, v) \in C\left([0, T] ; \tilde{H}^{s_{1}, s_{2}}\left(\mathbb{R}^{2}\right)\right) \times C\left([0, T] ; \tilde{H}^{s_{1}, s_{2}}\left(\mathbb{R}^{2}\right)\right) .
$$

Remark 2. Obviously the same result holds for any scalar KP-I or KP-II equation with "general" nonlinearity $f(u) u_{x}$, where $f$ is a smooth function. Note that $\tilde{H}^{s_{1}, s_{2}}\left(\mathbb{R}^{2}\right), s_{1}>3 / 2, s_{2}>1 / 2$ defines a larger class of possible initial data than the one considered in [17], where the data is supposed to be in a classical Sobolev space $H^{s}\left(\mathbb{R}^{2}\right), s>2$. The anisotropic Sobolev spaces seem to be a natural set for the initial data of KP type equations, since the scale invariance of these equations could be stated in terms of some anisotropic Sobolev spaces. The result of Theorem 2.1 does not depend on the sign of $a_{11} a_{22}-a_{12} a_{21}$ (i.e. does not distinguish the "KP-I" and "KP-II" case). In the next section, we will improve it a lot, in the "KP-II" case.

\section{Global Well posedness in $L^{2}\left(\gamma^{ \pm}>0\right)$}

\subsection{Preliminaries}

We first perform a scale change. Set

$$
w_{1}(t, x, y)=w^{+}\left(t, \frac{x}{\left(\gamma^{+}\right)^{\frac{1}{3}}}, \frac{y}{\left(\gamma^{+}\right)^{\frac{1}{6}}}\right), \quad w_{2}(t, x, y)=w^{-}\left(t, \frac{x}{\left(\gamma^{-}\right)^{\frac{1}{3}}}, \frac{y}{\left(\gamma^{-}\right)^{\frac{1}{6}}}\right) .
$$

Let $W=\left(w_{1}, w_{2}\right)^{t}$. Then $W$ satisfies

$$
\left(W_{t}+W_{x x x}+B(W) W_{x}\right)_{x}+W_{y y}=0,
$$

with

Write (9) in the form

$$
B(W)=\left(\begin{array}{cc}
c_{2} w_{1}+c_{1} w_{2} & c_{1} w_{1}+c_{3} w_{2} \\
c_{5} w_{1}+c_{4} w_{2} & c_{4} w_{1}+c_{6} w_{2}
\end{array}\right) .
$$

$$
i \partial_{t} W=p(D) W-i B(W) W_{x},
$$

where $D=\left(D_{1}, D_{2}\right), D_{1}=-i \partial_{x}, D_{2}=-i \partial_{y}$ and the operator $p(D)$ is defined through the Fourier transform

$$
\mathcal{F}(p(D) w)(\zeta)=p(\zeta) \mathcal{F}(w)(\zeta) .
$$

Here $\zeta=(\xi, \eta)$ stays for the Fourier variables corresponding to $(x, y)$ and

$$
p(\zeta)=-\xi^{3}+\frac{\eta^{2}}{\xi}
$$

Let $U(t)=\exp (-i t p(D))$ be the unitary group which generate the free evolution. We shall solve the integral equation corresponding to $(9)$

$$
\left.W(t)=U(t) W(0)-\int_{0}^{t} U\left(t-t^{\prime}\right) B\left(W\left(t^{\prime}\right)\right) W_{x}\left(t^{\prime}\right)\right) \mathrm{d} t^{\prime} .
$$


Let $\psi$ be a cut-off function such that

$$
\psi \in C_{0}^{\infty}(\mathbb{R}), \operatorname{supp} \psi \subset[-2,2], \psi=1 \text { over the interval }[-1,1]
$$

We consider a cut-off version of (11)

$$
\left.W(t)=\psi(t) U(t) W(0)-\psi(t) \int_{0}^{t} U\left(t-t^{\prime}\right) B\left(W\left(t^{\prime}\right)\right) W_{x}\left(t^{\prime}\right)\right) \mathrm{d} t^{\prime} .
$$

We shall solve globally in time (12) requiring smallness of the initial data. To the solutions of (12) will correspond local solutions of $(11)$ in the time interval $[-1,1]$. Then removing the smallness condition on the data we obtain solution of (11) in a small interval due to the scale invariance of the system. Now we construct the functional space where the solutions are expected to belong. Let $X_{s}$ be the space equipped with the Fourier transform restriction norm

$$
\|u\|_{X_{s}}=\left\|\langle\tau+p(\zeta)\rangle^{\frac{1}{2}}+\left(1+\frac{\langle\tau+p(\zeta)\rangle^{\frac{7}{32}}}{\langle\xi\rangle^{\frac{1}{4}}}\right)\langle\xi\rangle^{s} \widehat{u}(\tau, \zeta)\right\|_{L_{\tau, \zeta}^{2}} .
$$

The factor $\left(1+\frac{\langle\tau+p(\zeta)\rangle^{\frac{7}{32}}}{\langle\xi\rangle^{\frac{1}{4}}}\right)$ is not needed in the $\mathrm{KdV}$ case $(c f .[7,20])$ but it will be used here to deal with the low frequency cases in the bilinear estimate. It is clear that $X_{0} \subset C\left(\mathbb{R} ; L^{2}\left(\mathbb{R}^{2}\right)\right)$. Now we introduce the auxiliary spaces $Y_{s}$ equipped with the norm

$$
\|u\|_{Y_{s}}=\left\|\langle\tau+p(\zeta)\rangle^{-\frac{1}{2}+}\left(1+\frac{\langle\tau+p(\zeta)\rangle^{\frac{7}{32}}}{\langle\xi\rangle^{\frac{1}{4}}}\right)\langle\xi\rangle^{s} \widehat{u}(\tau, \zeta)\right\|_{L_{\tau, \zeta}^{2}} .
$$

For simplicity we set $X=X_{0}$ and $Y=Y_{0}$. Now we can formulate the estimates for the two terms in the right-hand side of (12).

Proposition 3.1. (linear estimates, $c f .[6,7,13])$. Let $w_{1,2}(0) \in L^{2}$. Then the following estimates hold

$$
\begin{gathered}
\left\|\psi(t) U(t) w_{1,2}(0)\right\|_{X} \leq c\left\|w_{1,2}(0)\right\|_{L^{2}}, \\
\left\|\psi(t) \int_{0}^{t} U\left(t-t^{\prime}\right) B\left(W\left(t^{\prime}\right)\right) W_{x}\left(t^{\prime}\right) \mathrm{d} t^{\prime}\right\|_{X_{s}} \leq c\left\{\left\|B\left(w_{1}, w_{2}\right)\right\|_{Y_{s}}+\left\|B\left(w_{1}, w_{1}\right)\right\|_{Y_{s}}+\left\|B\left(w_{2}, w_{2}\right)\right\|_{Y_{s}}\right\},
\end{gathered}
$$

where $B(u, v)=\partial_{x}(u v)$.

We shall solve (12) in $X$. Due to Proposition 3.1, in order to apply a fixed point argument, it is sufficient to estimate the $Y$ norm of the bilinear form $B(u, v)$ in terms of the $X$ norms of $u$ and $v$. We have the following Proposition which will be proved in the next section.

Proposition 3.2. Let $s \in\left[0, \frac{1}{29}\right)$. Then the following estimate holds

$$
\|B(u, v)\|_{Y_{s}} \leq c\|u\|_{X}\|v\|_{X}
$$

Using Propositions 3.1 and 3.2 we shall prove the following Theorem.

Theorem 3.1. For any $w_{1,2}(0) \in L^{2}\left(\mathbb{R}^{2}\right)$ there exists $T>0$ depending only on $\left\|w_{1,2}(0)\right\|_{L^{2}}$ and a unique solution $W=\left(w_{1}, w_{2}\right)^{t}$ of (11) in the time interval $[-T, T]$ satisfying

$$
w_{1,2} \in C\left([-T, T] ; L^{2}\left(\mathbb{R}^{2}\right)\right) \cap X
$$


Let $w_{1,2}$ be a solution of the integral equation (11). Then $w_{1,2}$ is a solution of (10) only if an additional restriction on the initial data is imposed. This is because of the singularity of $p(\zeta)$ at $\xi=0$. The operator $U(t)$ is defined for any $\phi \in L^{2}\left(\mathbb{R}^{2}\right)$ but $U(t) \phi$ has a time derivative which is a tempered distribution only if we suppose that formally $\phi$ has a "zero $x$ mean value". Actually $U(t) \phi$ has a well-defined time derivative provided $|\xi|^{-1} \widehat{\phi}(\xi, \eta) \in \mathcal{S}^{\prime}\left(\mathbb{R}^{2}\right)$. This condition means that formally $\int \phi(x, y) d x=0$. Furthermore due to the conservation of the $L^{2}$ the solutions can be extended globally in time. We are going to prove the following Theorem.

Theorem 3.2. Let

$$
w_{1,2}(0) \in L^{2}\left(\mathbb{R}^{2}\right) \text {, with }|\xi|^{-1} \mathcal{F}\left(w_{1,2}(0)\right)(\xi, \eta) \in \mathcal{S}^{\prime}\left(\mathbb{R}^{2}\right) .
$$

Then there exists a unique global solution $W=\left(w_{1}, w_{2}\right)^{t}$ of (10) satisfying

$$
w_{1,2} \in C\left(\mathbb{R} ; L^{2}\left(\mathbb{R}^{2}\right)\right) \cap X .
$$

Moreover the following decomposition of $W$ for any $t>0$ holds

$$
W(t)=U(t) W(0)+R(t)
$$

where $R(t) \in H^{s, 0}\left(\mathbb{R}^{2}\right) \times H^{s, 0}\left(\mathbb{R}^{2}\right)$, for $s \in\left[0, \frac{1}{29}\right)$.

To the solutions of (10) correspond solutions of (3), provided $\gamma^{ \pm}>0$. This last condition allows to perform the scale change reducing the diagonal matrix $\operatorname{diag}\left(\gamma^{+}, \gamma^{-}\right)$to the unit matrix. To the global solutions of (3) correspond global solutions of the original system (2). Note that $\gamma^{ \pm}>0$ is equivalent to $c_{n}^{2} c_{m}^{2} \lambda_{m n}^{2}<4 I_{m} I_{n} \lambda_{m} \lambda_{n}$ and therefore Theorem 1 is a direct consequence of Theorem 3.2.

\subsection{Proof of Proposition 3.2}

Set

$$
\sigma:=\sigma(\tau, \zeta)=\tau-\xi^{3}+\frac{\eta^{2}}{\xi}, \quad \sigma_{1}:=\sigma\left(\tau_{1}, \zeta_{1}\right), \quad \sigma_{2}:=\sigma\left(\tau-\tau_{1}, \zeta-\zeta_{1}\right) .
$$

Now we state an inequality which is actually the Strichartz estimate for KP injected into Bourgain's framework.

Proposition 3.3. Let $2 \leq q \leq 4$. Then for any $u \in L^{2}\left(\mathbb{R}^{3}\right)$ the following inequality holds

$$
\left\|\mathcal{F}^{-1}\left(\langle\sigma\rangle^{-b}|\hat{u}(\tau, \zeta)|\right)\right\|_{L^{q}} \leq c\|u\|_{L^{2}}
$$

where $b=2\left(1-\frac{2}{q}\right)\left(\frac{1}{2}+\right)$.

Proof. For any $\phi \in L^{2}\left(\mathbb{R}^{2}\right)$ the classical version of the Strichartz inequality for the KP equation (cf. [22], Prop. 2.3) yields

$$
\|U(t) \phi\|_{L^{4}} \leq c\|\phi\|_{L^{2}}
$$

Once we have (17), Lemma 3.3 of [13] gives for any $u \in X$

$$
\left\|\mathcal{F}^{-1}\left(\langle\sigma\rangle^{-\frac{1}{2}-}|\widehat{u}(\tau, \zeta)|\right)\right\|_{L^{4}} \leq c\|u\|_{L^{2}} .
$$

Interpolating between (18) and Plancherel identity completes the proof of Proposition 3.3. Now we state a Corollary of Proposition 3.3 which will be intensively used hereafter. 
Proposition 3.4. Let $\alpha_{1}, \alpha_{2}, \alpha_{3} \in\left[0, \frac{1}{2}+\varepsilon\right]$ and $\widehat{u}, \widehat{v}, \widehat{w}$ be positive. Then

$$
\int \frac{\widehat{u}\left(\tau_{1}, \zeta_{1}\right) \widehat{v}\left(\tau-\tau_{1}, \zeta-\zeta_{1}\right) \widehat{w}(\tau, \zeta)}{\left\langle\sigma_{1}\right\rangle^{\alpha_{1}}\left\langle\sigma_{2}\right\rangle^{\alpha_{2}}\langle\sigma\rangle^{\alpha_{3}}} \mathrm{~d} \tau \mathrm{d} \zeta \mathrm{d} \tau_{1} \mathrm{~d} \zeta_{1} \leq c\|u\|_{L^{2}}\|v\|_{L^{2}}\|w\|_{L^{2}}
$$

provided $\alpha_{1}+\alpha_{2}+\alpha_{3} \geq 1+2 \varepsilon$.

Proof. We denote by $I$ the left-hand side of (19). Clearly we can assume that $\alpha_{1}+\alpha_{2}+\alpha_{3}=1+2 \varepsilon$ Then Hölder inequality, Plancherel identity and Proposition 3.3 yield

$$
\begin{aligned}
I & \leq\left\|\mathcal{F}^{-1}\left(\left\langle\sigma_{1}\right\rangle^{-\alpha_{1}} \widehat{u}\right)\right\|_{L^{q_{1}}}\left\|\mathcal{F}^{-1}\left(\left\langle\sigma_{2}\right\rangle^{-\alpha_{2}} \widehat{v}\right)\right\|_{L^{q_{2}}}\left\|\mathcal{F}^{-1}\left(\langle\sigma\rangle^{-\alpha} \widehat{w}\right)\right\|_{L^{q_{3}}} \\
& \leq c\|u\|_{L^{2}}\|v\|_{L^{2}}\|w\|_{L^{2}},
\end{aligned}
$$

provided $\alpha_{j}=2\left(1-\frac{2}{q_{j}}\right)\left(\frac{1}{2}+\varepsilon\right), j=1,2,3$ and $\frac{1}{q_{1}}+\frac{1}{q_{2}}+\frac{1}{q_{3}}=1$. But the last equality is equivalent to $\alpha_{1}+\alpha_{2}+\alpha_{3}=1+2 \varepsilon$ which completes the proof of Proposition 3.4.

We shall also make use of the following calculus inequalities.

Proposition 3.5. For any $a \in \mathbb{R}$ the following inequalities hold

$$
\begin{gathered}
\int_{-\infty}^{\infty} \frac{\mathrm{d} t}{\langle t\rangle^{1 \pm}\langle t-a\rangle^{1+}} \leq \frac{c}{\langle a\rangle^{1 \pm}} \\
\int_{-\infty}^{\infty} \frac{\mathrm{d} t}{\langle t\rangle^{1+}|t-a|^{\frac{1}{2}}} \leq \frac{c}{\langle a\rangle^{\frac{1}{2}}} .
\end{gathered}
$$

Set

$$
\theta:=\theta(\tau, \zeta)=\frac{\langle\sigma\rangle^{\frac{7}{32}}}{\langle\xi\rangle^{1 / 4}}, \quad \theta_{1}=\theta\left(\tau_{1}, \zeta_{1}\right), \quad \theta_{2}=\theta\left(\tau-\tau_{1}, \zeta-\zeta_{1}\right)
$$

A duality argument shows that (15) is equivalent to

$$
\left|\iint K\left(\tau, \zeta, \tau_{1}, \zeta_{1}\right) \widehat{u}\left(\tau_{1}, \zeta_{1}\right) \widehat{v}\left(\tau-\tau_{1}, \zeta-\zeta_{1}\right) \widehat{w}(\tau, \zeta) \mathrm{d} \tau_{1} \mathrm{~d} \zeta_{1} \mathrm{~d} \tau \mathrm{d} \zeta\right| \leq c\|u\|_{L^{2}}\|v\|_{L^{2}}\|w\|_{L^{2}}
$$

where

$$
K\left(\tau, \zeta, \tau_{1}, \zeta_{1}\right)=\frac{|\xi|\langle\xi\rangle^{s}\langle\theta\rangle}{\langle\sigma\rangle^{\frac{1}{2}-}\left\langle\sigma_{1}\right\rangle^{\frac{1}{2}+}\left\langle\sigma_{2}\right\rangle^{\frac{1}{2}+}\left\langle\theta_{1}\right\rangle\left\langle\theta_{2}\right\rangle}
$$

Without loss of generality we can assume that $\widehat{u} \geq 0, \widehat{v} \geq 0$ and $\widehat{w} \geq 0$. By symmetry we can assume that $\left|\sigma_{1}\right| \geq\left|\sigma_{2}\right|$. To gain the loss of a derivative in the nonlinear term we shall use the relation (cf. [8])

$$
\sigma_{1}+\sigma_{2}-\sigma=3 \xi_{1} \xi\left(\xi-\xi_{1}\right)+\frac{\left(\xi_{1} \eta-\xi \eta_{1}\right)^{2}}{\xi_{1} \xi\left(\xi-\xi_{1}\right)}
$$

and hence

$$
\max \left\{|\sigma|,\left|\sigma_{1}\right|,\left|\sigma_{2}\right|\right\} \geq\left|\xi_{1} \xi\left(\xi-\xi_{1}\right)\right|
$$


Let $J$ we be the left-hand side of (22). We consider several regions for $\left(\tau, \zeta, \tau_{1}, \zeta_{1}\right)$.

Case 1. $|\xi| \leq c_{0}$, where $c_{0}$ is a sufficiently large constant. We denote by $J_{1}$ the restriction of $J$ on this region. Using that $\langle\theta\rangle \leq c\langle\sigma\rangle^{\frac{1}{2}-}$ we obtain

$$
K\left(\tau, \zeta, \tau_{1}, \zeta_{1}\right) \leq \frac{c}{\left\langle\sigma_{1}\right\rangle^{\frac{1}{2}+}\left\langle\sigma_{2}\right\rangle^{\frac{1}{2}+}}
$$

It suffices to use Proposition 3.4 to get a bound for $J_{1}$.

Case 2. $|\sigma| \geq\left|\sigma_{1}\right|, \quad|\xi| \geq c_{0}$. We denote by $J_{2}$ the restriction of $J$ on this region. Using Cauchy-Schwarz inequality and (20) we obtain

$$
J_{2} \leq \int I(\tau, \zeta)\left\{\int_{|\sigma| \geq\left|\sigma_{1}\right|}\left|\widehat{u}\left(\tau_{1}, \zeta_{1}\right) \widehat{v}\left(\tau-\tau_{1}, \zeta-\zeta_{1}\right)\right|^{2} \mathrm{~d} \tau_{1} \mathrm{~d} \zeta_{1}\right\}^{\frac{1}{2}} \widehat{w}(\tau, \zeta) \mathrm{d} \tau \mathrm{d} \zeta
$$

where via (20)

$$
I(\tau, \zeta)=\frac{|\xi|\langle\xi\rangle^{s}\langle\theta\rangle}{\langle\sigma\rangle^{\frac{1}{2}-}}\left(\int_{|\sigma| \geq\left|\sigma_{1}\right|} \frac{\mathrm{d} \tau_{1} \mathrm{~d} \zeta_{1}}{\left\langle\sigma_{1}\right\rangle^{1+}\left\langle\sigma_{2}\right\rangle^{1+}\left\langle\theta_{1}\right\rangle^{2}\left\langle\theta_{2}\right\rangle^{2}}\right)^{\frac{1}{2}} \leq \frac{\langle\xi\rangle^{1+s}\langle\theta\rangle}{\langle\sigma\rangle^{\frac{1}{2}-}}\left(\int \frac{\mathrm{d} \zeta_{1}}{\left\langle\sigma_{1}+\sigma_{2}\right\rangle^{1+}}\right)^{\frac{1}{2}} .
$$

We perform a change of variables similar to [18]

$$
\alpha=\sigma_{1}+\sigma_{2}, \quad \beta=3 \xi \xi_{1}\left(\xi-\xi_{1}\right) .
$$

Note that

$$
\beta \in\left[-3|\sigma|, \min \left\{3 / 4 \xi^{3}, 3|\sigma|\right\}\right], \quad \text { when } \xi \geq 0
$$

and

$$
\left.\beta \in\left[\max \left\{3 / 4 \xi^{3},-3|\sigma|\right\}, 3|\sigma|\right\}\right], \quad \text { when } \xi \leq 0 .
$$

We assume that $\xi \geq 0$. If $\xi \leq 0$ then the arguments are similar. We have that

$$
\mathrm{d} \zeta_{1}=\frac{c|\beta|^{\frac{1}{2}} \mathrm{~d} \alpha \mathrm{d} \beta}{|\xi|^{\frac{3}{2}}\left(\frac{3}{4} \xi^{3}-\beta\right)^{\frac{1}{2}}|\sigma+\beta-\alpha|^{\frac{1}{2}}}
$$

Therefore we obtain using (21)

$$
\begin{aligned}
I(\tau, \zeta) & \leq \frac{c\langle\xi\rangle^{\frac{1}{4}+s}\langle\theta\rangle}{\langle\sigma\rangle^{\frac{1}{2}-}}\left\{\int_{-3|\sigma|}^{\min \left\{3 / 4 \xi^{3}, 3|\sigma|\right\}} \int_{-\infty}^{\infty} \frac{|\beta|^{\frac{1}{2}} \mathrm{~d} \alpha \mathrm{d} \beta}{\left(\frac{3}{4} \xi^{3}-\beta\right)^{\frac{1}{2}}|\sigma+\beta-\alpha|^{\frac{1}{2}}\langle\alpha\rangle^{1+}}\right\}^{\frac{1}{2}} \\
& \leq \frac{c\langle\xi\rangle^{\frac{1}{4}+s}\langle\theta\rangle}{\langle\sigma\rangle^{\frac{1}{2}-}}\left\{\int_{-3|\sigma|}^{\min \left\{3 / 4 \xi^{3}, 3|\sigma|\right\}} \frac{|\beta|^{\frac{1}{2}} \mathrm{~d} \beta}{\left(\frac{3}{4} \xi^{3}-\beta\right)^{\frac{1}{2}}\langle\sigma+\beta\rangle^{\frac{1}{2}}}\right\}^{\frac{1}{2}} .
\end{aligned}
$$

Consider two cases for $(\tau, \zeta)$. 
- $\frac{3}{4}|\xi|^{3} \leq 4|\sigma|$. We have that

$$
\begin{aligned}
I(\tau, \zeta) & \leq \frac{c\langle\xi\rangle^{\frac{1}{4}+s}\langle\theta\rangle}{\langle\sigma\rangle^{\frac{1}{2}-}}\left\{\int_{-3|\sigma|}^{0} \cdots+\int_{0}^{\frac{3}{4} \xi^{3}} \cdots\right\}^{\frac{1}{2}} \\
& \leq \frac{c\langle\xi\rangle^{\frac{1}{4}+s}\langle\theta\rangle}{\langle\sigma\rangle^{\frac{1}{2}-}}\left(\langle\sigma\rangle^{\frac{1}{2}}+\xi^{\frac{3}{2}} \ln (1+\langle\sigma\rangle)\right)^{\frac{1}{2}} \\
& \leq \frac{c\langle\xi\rangle^{\frac{1}{4}+s}\langle\theta\rangle}{\langle\sigma\rangle^{\frac{1}{4}-}}+\frac{c\langle\xi\rangle\langle\theta\rangle}{\langle\sigma\rangle^{\frac{1}{2}-}} .
\end{aligned}
$$

Let $|\sigma|^{\frac{7}{32}} \leq|\xi|^{1 / 4}$. Then we have

$$
I(\tau, \zeta) \leq \frac{1}{\langle\xi\rangle^{\frac{1}{2}-s-}} \leq \text { const. }
$$

Let $|\sigma|^{\frac{7}{32}} \geq|\xi|^{1 / 4}$. It follows

$$
I(\tau, \zeta) \leq \frac{1}{\langle\xi\rangle^{\frac{3}{32}-s-}} \leq \text { const. }
$$

- $4|\sigma| \leq \frac{3}{4}|\xi|^{3}$. In this case we have

$$
\begin{aligned}
I(\tau, \zeta) & \leq \frac{c\langle\xi\rangle^{\frac{1}{4}+s}\langle\theta\rangle}{\langle\sigma\rangle^{\frac{1}{4}-}}\left\{\int_{-3|\sigma|}^{3|\sigma|} \frac{\mathrm{d} \beta}{\left(\frac{3}{4} \xi^{3}-\beta\right)^{\frac{1}{2}}\langle\sigma+\beta\rangle^{\frac{1}{2}}}\right\}^{\frac{1}{2}} \\
& \leq \frac{c\langle\theta\rangle}{\langle\sigma\rangle^{\frac{1}{4}-}\langle\xi\rangle^{\frac{1}{2}-s}}\left\{\int_{-3|\sigma|}^{3|\sigma|} \frac{\mathrm{d} \beta}{\langle\sigma+\beta\rangle^{\frac{1}{2}}}\right\}^{\frac{1}{2}} \\
& \leq \frac{c\langle\theta\rangle\langle\sigma\rangle^{0+}}{\langle\xi\rangle^{\frac{1}{2}-s}} .
\end{aligned}
$$

Let $|\sigma|^{\frac{7}{32}} \leq|\xi|^{\frac{1}{4}}$. Then we have

$$
I(\tau, \zeta) \leq \frac{c}{\langle\xi\rangle^{\frac{1}{2}-s-}} \leq \text { const. }
$$

Let $|\sigma|^{\frac{7}{32}} \geq|\xi|^{\frac{1}{4}}$. Then we have

$$
I(\tau, \zeta) \leq \frac{c\langle\sigma\rangle^{\frac{7}{32}+}}{\langle\xi\rangle^{\frac{3}{4}-s}} \leq \frac{c}{\langle\xi\rangle^{\frac{3}{32}-s-}} \leq \text { const. }
$$

Hence $I(\tau, \zeta)$ is bounded and the use of Cauchy-Schwarz inequality yields

$$
J_{2} \leq c\|u\|_{L^{2}}\|v\|_{L^{2}}\|w\|_{L^{2}} .
$$


Case 3. $\left|\sigma_{1}\right| \geq|\sigma|, \quad|\xi| \geq c_{0}, \quad\left|\xi_{1}\right| \leq 1$. We denote by $J_{3}$ the restriction of $J$ on this region. We shall estimate $J_{3}$ by a localization with respect to $\xi_{1}$ and $\left\langle\sigma_{1}\right\rangle$. Set

$$
J_{3}^{K M}=\iint_{A^{K M}} K\left(\tau, \zeta, \tau_{1}, \zeta_{1}\right) \widehat{u}\left(\tau_{1}, \zeta_{1}\right) \widehat{v}\left(\tau-\tau_{1}, \zeta-\zeta_{1}\right) \widehat{w}(\tau, \zeta) \mathrm{d} \tau_{1} \mathrm{~d} \zeta_{1} \mathrm{~d} \tau \mathrm{d} \zeta,
$$

where

$$
A^{K M}=\left\{\left(\tau_{1}, \zeta_{1}\right):\left|\xi_{1}\right| \sim M,\left\langle\sigma_{1}\right\rangle \sim K\right\} .
$$

We have

$$
J_{3} \leq \sum_{K, M} J_{3}^{K M},
$$

where the sum is taken over $K=2^{k}, k=0,1,2, \ldots$ and $M=2^{m}, m=0,-1,-2, \ldots$ Note that in this case (24) implies

$$
|\xi| \leq c K^{\frac{1}{2}} M^{-\frac{1}{2}} .
$$

Now we shall estimate $J_{3}^{K M}$ in two ways. First by a direct estimate for $K$ we bound $J_{3}^{K M}$. Then we estimate $J_{3}^{K M}$ by the aid of Proposition 3.4. A suitable interpolation provides the needed inequality. Cauchy-Schwarz inequality yields

$$
J_{3}^{K M} \leq \int_{\mathbb{R}^{3}} I^{K M}(\tau, \zeta)\left\{\int_{A^{K M}}\left|\widehat{u}\left(\tau_{1}, \zeta_{1}\right) \widehat{v}\left(\tau-\tau_{1}, \zeta-\zeta_{1}\right)\right|^{2} \mathrm{~d} \tau_{1} \mathrm{~d} \zeta_{1}\right\}^{\frac{1}{2}} \widehat{w}(\tau, \zeta) \mathrm{d} \tau \mathrm{d} \zeta,
$$

where

$$
I^{K M}(\tau, \zeta)=\frac{|\xi|^{1+s}\langle\theta\rangle}{\langle\sigma\rangle^{\frac{1}{2}-}}\left(\int_{A^{K M}} \frac{\mathrm{d} \tau_{1} \mathrm{~d} \zeta_{1}}{\left\langle\sigma_{1}\right\rangle^{1+}\left\langle\sigma_{2}\right\rangle^{1+}\left\langle\theta_{1}\right\rangle^{2}\left\langle\theta_{2}\right\rangle^{2}}\right)^{\frac{1}{2}}
$$

We change the variables

$$
\alpha=\sigma_{1}+\sigma_{2}, \quad \beta=3 \xi \xi_{1}\left(\xi-\xi_{1}\right) .
$$

As in Case 2 we suppose that $\xi \geq 0$. We have

$$
|\beta| \leq 3|\xi|(|\xi|+1) 2 M \leq c|\xi|^{2} M .
$$

Hence using that $\langle\theta\rangle \leq c\langle\sigma\rangle^{\frac{1}{2}-}$ we obtain

$$
I^{K M} \leq\left\{\int_{-\infty}^{\infty} \int_{-\infty}^{\infty} \int_{-c M|\xi|^{2}}^{c M|\xi|^{2}} \frac{|\xi|^{\frac{1}{2}+2 s}|\beta|^{\frac{1}{2}} \mathrm{~d} \tau_{1} \mathrm{~d} \alpha \mathrm{d} \beta}{\left\langle\sigma_{1}\right\rangle^{1+}\left\langle\alpha-\sigma_{1}\right\rangle^{1+}\left(\frac{3}{4} \xi^{3}-\beta\right)^{\frac{1}{2}}|\sigma+\beta-\alpha|^{\frac{1}{2}}\left\langle\theta_{1}\right\rangle^{2}}\right\}^{\frac{1}{2}} .
$$

Since $|\beta| \leq c\left|\sigma_{1}\right|$ we obtain

Hence

$$
\frac{|\beta|^{\frac{1}{2}}}{\left\langle\theta_{1}\right\rangle^{2}} \leq c\left\langle\sigma_{1}\right\rangle^{\frac{1}{16}} \leq c K^{\frac{1}{16}}
$$

$$
\begin{aligned}
I^{K M}(\tau, \zeta) & \leq c|\xi|^{\frac{1}{4}+s} K^{\frac{1}{32}}\left\{\int_{-\infty}^{\infty} \int_{-c M|\xi|^{2}}^{c M|\xi|^{2}} \frac{\mathrm{d} \alpha \mathrm{d} \beta}{\langle\alpha\rangle^{1+}\left(\frac{3}{4} \xi^{3}-\beta\right)^{\frac{1}{2}}|\sigma+\beta-\alpha|^{\frac{1}{2}}}\right\}^{\frac{1}{2}} \\
& \leq c|\xi|^{\frac{1}{4}+s} K^{\frac{1}{32}}\left\{\int_{-c M|\xi|^{2}}^{c M|\xi|^{2}} \frac{\mathrm{d} \beta}{\left(\frac{3}{4} \xi^{3}-\beta\right)^{\frac{1}{2}}|\sigma+\beta|^{\frac{1}{2}}}\right\}^{\frac{1}{2}} .
\end{aligned}
$$


Since $|\xi| \geq c_{0}$, for sufficiently large $c_{0}$ we have $\left|\frac{3}{4} \xi^{3}-\beta\right| \geq \frac{3}{8} \xi^{3}$ and therefore

$$
\begin{aligned}
I^{K M}(\tau, \zeta) & \leq \frac{c K^{\frac{1}{32}}}{|\xi|^{\frac{1}{2}-s}}\left\{\int_{-c M|\xi|^{2}}^{c M|\xi|^{2}} \frac{\mathrm{d} \beta}{|\sigma+\beta|^{\frac{1}{2}}}\right\}^{\frac{1}{2}} \\
& \leq c K^{\frac{1}{32}+\frac{s}{2}} M^{\frac{1}{4}-\frac{s}{2}},
\end{aligned}
$$

Cauchy-Schwarz inequality yields

$$
J_{3}^{K M} \leq c K^{\frac{1}{32}+\frac{s}{2}} M^{\frac{1}{4}-\frac{s}{2}}\|u\|_{L^{2}}\|v\|_{L^{2}}\|w\|_{L^{2}} .
$$

Now we shall estimate $J_{3}^{K M}(\tau, \zeta)$ by the aid of Proposition 3.4. We have

$$
K\left(\tau, \zeta, \tau_{1}, \zeta_{1}\right) \leq \frac{|\xi|^{1+s}\langle\theta\rangle}{\langle\sigma\rangle^{\frac{1}{2}-}\left\langle\sigma_{1}\right\rangle^{\frac{23}{32}+}\left\langle\sigma_{2}\right\rangle^{\frac{1}{2}+}}
$$

Let $|\sigma|^{\frac{7}{32}} \leq|\xi|^{\frac{1}{4}}$. We denote by $J_{31}^{K M}$ the restriction of $J_{3}^{K M}$ on this region. We have

$$
K\left(\tau, \zeta, \tau_{1}, \zeta_{1}\right) \leq \frac{c M^{-\frac{1+s}{2}} K^{-\frac{1}{8}+\frac{s}{2}+}}{\langle\sigma\rangle^{\frac{1}{2}-}\left\langle\sigma_{1}\right\rangle^{\frac{3}{32}}+\left\langle\sigma_{2}\right\rangle^{\frac{1}{2}+}}
$$

Using Proposition 3.4 we obtain

$$
J_{31}^{K M} \leq \frac{c}{M^{\frac{1+s}{2}} K^{\frac{1}{8}-\frac{s}{2}-}}\|u\|_{L^{2}}\|v\|_{L^{2}}\|w\|_{L^{2}} .
$$

Let $|\sigma|^{\frac{7}{32}} \geq|\xi|^{\frac{1}{4}}$. We denote by $J_{32}^{K M}$ the restriction of $J_{3}^{K M}$ on this region. We have

$$
K\left(\tau, \zeta, \tau_{1}, \zeta_{1}\right) \leq \frac{c M^{-\frac{3+4 s}{8}} K^{-\frac{1}{8}+\frac{s}{2}+}}{\langle\sigma\rangle^{\frac{9}{32}-}\left\langle\sigma_{1}\right\rangle^{\frac{7}{32}}+\left\langle\sigma_{2}\right\rangle^{\frac{1}{2}+}}
$$

Using Proposition 3.4 we obtain

$$
J_{32}^{K M} \leq \frac{c}{M^{\frac{3+4 s}{8}} K^{\frac{1}{8}-\frac{s}{2}-}}\|u\|_{L^{2}}\|v\|_{L^{2}}\|w\|_{L^{2}} .
$$

Further we have

$$
J_{3}^{K M} \leq J_{31}^{K M}+J_{32}^{K M} \leq M^{-\frac{1}{2}-\frac{s}{2}} K^{-\frac{1}{8}+\frac{s}{2}+}\|u\|_{L^{2}}\|v\|_{L^{2}}\|w\|_{L^{2}} .
$$

Interpolation between (25) and (26) with weights $\frac{2}{3}+\frac{2 s}{3}+$ and $\frac{1}{3}-\frac{2 s}{3}-$ respectively yields

$$
J_{3}^{K M} \leq \frac{M^{\alpha_{1}}}{K^{\alpha_{2}}}\|u\|_{L^{2}}\|v\|_{L^{2}}\|w\|_{L^{2}}
$$

For

$$
\alpha_{1}=\left(\frac{2}{3}+\frac{2 s}{3}+\right)\left(\frac{1}{4}-\frac{s}{2}\right)+\left(\frac{1}{3}-\frac{2 s}{3}-\right)\left(-\frac{1}{2}-\frac{s}{2}\right)=0+
$$


and

$$
\alpha_{2}=\left(\frac{2}{3}+\frac{2 s}{3}+\right)\left(-\frac{1}{32}-\frac{s}{2}\right)+\left(\frac{1}{3}-\frac{2 s}{3}-\right)\left(\frac{1}{8}-\frac{s}{2}-\right)=\frac{1-29 s}{48}-
$$

Hence since $s>1 / 29$ we obtain that $\alpha_{1}$ and $\alpha_{2}$ are positive. Summing over $K$ and $M$ yields

$$
J_{3} \leq c\|u\|_{L^{2}}\|v\|_{L^{2}} \mid w \|_{L^{2}} .
$$

Case 4. $\left|\sigma_{1}\right| \geq|\sigma|, \quad|\xi| \geq c_{0}, \quad|\xi| \leq 2\left|\xi_{1}\right|$. We denote by $J_{4}$ the restriction of $J$ on this region. In this case we have

$$
\frac{\langle\theta\rangle}{\left\langle\theta_{1}\right\rangle} \leq c\left|\xi_{1}\right|^{\frac{1}{4}}
$$

Hence

$$
K\left(\tau, \zeta, \tau_{1}, \zeta_{1}\right) \leq \frac{c\left|\xi_{1}\right|^{\frac{5}{4}+s}}{\langle\sigma\rangle^{\frac{1}{2}-}\left\langle\sigma_{1}\right\rangle^{\frac{1}{2}+}\left\langle\sigma_{2}\right\rangle^{\frac{1}{2}+}}
$$

Using Cauchy-Schwarz inequality we obtain

$$
J_{4} \leq \int I_{1}\left(\tau_{1}, \zeta_{1}\right)\left\{\int_{\left|\sigma_{1}\right| \geq|\sigma|}\left|\widehat{w}(\tau, \zeta) \widehat{v}\left(\tau-\tau_{1}, \zeta-\zeta_{1}\right)\right|^{2} \mathrm{~d} \tau \mathrm{d} \zeta\right\}^{\frac{1}{2}} \widehat{u}\left(\tau_{1}, \zeta_{1}\right) \mathrm{d} \tau_{1} \mathrm{~d} \zeta_{1},
$$

where

$$
\begin{aligned}
I_{1}\left(\tau_{1}, \zeta_{1}\right) & =\frac{\left|\xi_{1}\right|^{\frac{5}{4}+s}}{\left\langle\sigma_{1}\right\rangle^{\frac{1}{2}+}}\left(\int_{\left|\sigma_{1}\right| \geq|\sigma|} \frac{\mathrm{d} \tau \mathrm{d} \zeta}{\langle\sigma\rangle^{1+}\left\langle\sigma_{2}\right\rangle^{1+}}\right)^{\frac{1}{2}} \\
& \leq \frac{c\left|\xi_{1}\right|^{\frac{5}{4}+s}}{\left\langle\sigma_{1}\right\rangle^{\frac{1}{2}-}}\left(\int \frac{\mathrm{d} \zeta}{\left\langle\sigma-\sigma_{2}\right\rangle^{1+}}\right)^{\frac{1}{2}}
\end{aligned}
$$

where we used that $\left|\sigma_{1}\right|$ dominates in this case. We perform a change of variables

$$
\alpha=\sigma-\sigma_{2}, \quad \beta=3 \xi \xi_{1}\left(\xi_{1}-\xi\right) .
$$

We can assume that $\xi_{1} \geq 0$. If $\xi_{1} \leq 0$ then the arguments are similar. Further we have

$$
\mathrm{d} \zeta=\frac{c|\beta|^{\frac{1}{2}} \mathrm{~d} \alpha \mathrm{d} \beta}{\left|\xi_{1}\right|^{3 / 2}\left(\frac{3}{4} \xi_{1}^{3}-\beta\right)^{\frac{1}{2}}\left|\sigma_{1}+\beta-\alpha\right|^{\frac{1}{2}}} .
$$

Therefore we obtain using (21)

$$
I_{1}\left(\tau_{1}, \zeta_{1}\right) \leq \frac{c\left|\xi_{1}\right|^{\frac{1}{2}+s}}{\left\langle\sigma_{1}\right\rangle^{\frac{1}{2}-}}\left\{\int_{-3\left|\sigma_{1}\right|}^{\min \left\{3 / 4 \xi_{1}^{3}, 3\left|\sigma_{1}\right|\right\}} \frac{|\beta|^{\frac{1}{2}} \mathrm{~d} \beta}{\left(\frac{3}{4} \xi_{1}^{3}-\beta\right)^{\frac{1}{2}}\left\langle\sigma_{1}+\beta\right\rangle^{\frac{1}{2}}}\right\}^{\frac{1}{2}} .
$$

Now we consider two cases for $\left(\tau_{1}, \zeta_{1}\right)$. 
- $\frac{3}{4}\left|\xi_{1}\right|^{3} \leq 4\left|\sigma_{1}\right|$. We have

$$
\begin{aligned}
I_{1}\left(\tau_{1}, \zeta_{1}\right) & \leq \frac{c\left|\xi_{1}\right|^{\frac{1}{2}+s}}{\left\langle\sigma_{1}\right\rangle^{\frac{1}{2}}-}\left\{\int_{-3\left|\sigma_{1}\right|}^{0} \cdots+\int_{0}^{\frac{3}{4} \xi_{1}^{3}} \cdots\right\}^{\frac{1}{2}} \\
& \leq \frac{c\left|\xi_{1}\right|^{\frac{1}{2}+s}}{\left\langle\sigma_{1}\right\rangle^{\frac{1}{2}}-}\left\{\left\langle\sigma_{1}\right\rangle^{\frac{1}{2}}+\xi_{1}^{\frac{3}{2}} \ln \left(1+\left\langle\sigma_{1}\right\rangle\right)\right\}^{\frac{1}{2}} \\
& \leq \frac{c}{\left|\xi_{1}\right|^{\frac{1}{4}-s-0}} \leq \text { const. }
\end{aligned}
$$

- $4\left|\sigma_{1}\right| \leq \frac{3}{4}\left|\xi_{1}\right|^{3}$. In this case we have

$$
\begin{aligned}
I_{1}\left(\tau_{1}, \zeta_{1}\right) & \leq \frac{c\left|\xi_{1}\right|^{\frac{1}{2}+s}}{\left\langle\sigma_{1}\right\rangle^{\frac{1}{4}-}}\left\{\int_{-3\left|\sigma_{1}\right|}^{3\left|\sigma_{1}\right|} \frac{d \beta}{\left(\frac{3}{4} \xi_{1}^{3}-\beta\right)^{\frac{1}{2}}\left\langle\sigma_{1}+\beta\right\rangle^{\frac{1}{2}}}\right\}^{\frac{1}{2}} \\
& \leq \frac{c}{\left\langle\sigma_{1}\right\rangle^{\frac{1}{4}-}\left|\xi_{1}\right|^{\frac{1}{4}-s}}\left\{\int_{-3\left|\sigma_{1}\right|}^{3\left|\sigma_{1}\right|} \frac{d \beta}{\left\langle\sigma_{1}+\beta\right\rangle^{\frac{1}{2}}}\right\}^{\frac{1}{2}} \\
& \leq \frac{c\left\langle\sigma_{1}\right\rangle^{0+}}{\left|\xi_{1}\right|^{\frac{1}{4}-s}} \leq \text { const. }
\end{aligned}
$$

Hence $I_{1}$ is bounded. Therefore we obtain by Cauchy-Schwarz inequality

$$
J_{4} \leq c\|u\|_{L^{2}}\|v\|_{L^{2}}\|w\|_{L^{2}}
$$

Case 5. $\left|\sigma_{1}\right| \geq|\sigma|, \quad|\xi| \geq c_{0}, \quad|\xi| \geq 2\left|\xi_{1}\right|, \quad\left|\xi_{1}\right| \geq 1$. We denote by $J_{5}$ the restriction of $J$ on this region. In this case we have that $|\xi| \leq 2\left|\xi-\xi_{1}\right|$.

Let $|\sigma|^{\frac{7}{32}} \leq|\xi|^{\frac{1}{4}}$. We denote by $J_{51}$ the restriction of $J_{5}$ on this region. We have

$$
K\left(\tau, \zeta, \tau_{1}, \zeta_{1}\right) \leq \frac{c|\xi|\left|\xi_{1}\right|^{\frac{1}{4}}}{\langle\sigma\rangle^{\frac{1}{2}-}\left\langle\sigma_{1}\right\rangle^{\frac{23}{32}}+\left\langle\sigma_{2}\right\rangle^{\frac{1}{2}+}} \leq \frac{c}{\langle\sigma\rangle^{\frac{1}{2}-}\left\langle\sigma_{1}\right\rangle^{\frac{3}{32}}\left\langle\sigma_{2}\right\rangle^{\frac{1}{2}+}} .
$$

Proposition 3.4 yields

$$
J_{51} \leq c\|u\|_{L^{2}}\|v\|_{L^{2}}\|w\|_{L^{2}} .
$$

Let $|\sigma|^{\frac{7}{32}} \geq|\xi|^{\frac{1}{4}}$. We denote by $J_{52}$ the restriction of $J_{5}$ on this region. We have

$$
K\left(\tau, \zeta, \tau_{1}, \zeta_{1}\right) \leq \frac{c|\xi|^{\frac{3}{4}}\left|\xi_{1}\right|^{\frac{1}{4}}}{\langle\sigma\rangle^{\frac{9}{32}}-\left\langle\sigma_{1}\right\rangle^{\frac{23}{32}}\left\langle\sigma_{2}\right\rangle^{\frac{1}{2}+}}
$$

and

$$
|\xi|^{\frac{3}{4}}\left|\xi_{1}\right|^{\frac{1}{4}} \leq c|\xi|^{\frac{1}{2}}\left|\xi_{1}\right|^{\frac{1}{4}}\left|\xi-\xi_{1}\right|^{\frac{1}{4}} \leq c\left\langle\sigma_{1}\right\rangle^{\frac{1}{4}}\left\langle\sigma_{1}\right\rangle^{\frac{1}{8}}=c\left\langle\sigma_{1}\right\rangle^{\frac{3}{8}} .
$$


Therefore

$$
K\left(\tau, \zeta, \tau_{1}, \zeta_{1}\right) \leq \frac{c}{\langle\sigma\rangle^{\frac{9}{32}}-\left\langle\sigma_{1}\right\rangle^{\frac{11}{32}+}\left\langle\sigma_{2}\right\rangle^{\frac{1}{2}+}}
$$

Proposition 3.4 yields

$$
J_{52} \leq c\|u\|_{L^{2}}\|v\|_{L^{2}}\|w\|_{L^{2}} .
$$

This completes the proof of Proposition 3.2.

\subsection{Proof of Theorem 3.1}

Let

$$
L: X \times X \longmapsto X \times X
$$

is an operator defined as

$$
L(W)=U(t) W(0)-\int_{0}^{t} U\left(t-t^{\prime}\right) B\left(W\left(t^{\prime}\right)\right) W_{x}\left(t^{\prime}\right) \mathrm{d} t^{\prime}
$$

Then Propositions 1 and 2 yield

$$
\begin{gathered}
\|\psi(t) L(W)\|_{X \times X} \leq c\left\{\left\|w_{1}(0)\right\|_{L^{2}}+\left\|w_{2}(0)\right\|_{L^{2}}+\left\|w_{1}\right\|_{X}^{2}+\left\|w_{2}\right\|_{X}^{2}\right\} \\
\left\|\psi(t) L\left(W_{1}\right)-\psi(t) L\left(W_{2}\right)\right\|_{X \times X} \leq c\left\|W_{1}-W_{2}\right\|_{X \times X}\left\|W_{1}+W_{2}\right\|_{X \times X} .
\end{gathered}
$$

A standard fixed point argument provides a fixed point of $\psi(t) L$ for $\left\|w_{1,2}(0)\right\|_{L^{2}}$ sufficiently small. To prove local existence for arbitrary data in $w_{1,2}(0) \in L^{2}$ we shall perform a scaling argument. If $u(t, x, y)$ is a function then we set

$$
u_{T}(t, x, y)=T^{2 / 3} u\left(T t, T^{1 / 3} x, T^{2 / 3} y\right) .
$$

Similarly if $\phi(x, y)$ is a function then

$$
\phi_{T}(x, y)=T^{2 / 3} \phi\left(T^{1 / 3} x, T^{2 / 3} y\right)
$$

Note that if $u(t, x, y)$ is a solution of $\mathrm{KP}$ equation with data $\phi_{T}(x, y)$ on $[-1,1]$ then so is $u_{T^{-1}}(t, x, y)$ with data $\phi(x, y)$ on $[-T, T]$. We have that $\left\|\phi_{T}\right\|_{L^{2}}=T^{1 / 6}\|\phi\|_{L^{2}}$. Hence if $T$ is sufficiently small then the equation $W=L(W)$ has a solution on $[-T, T]$. This proves the existence. For the proof of the uniqueness we refer to $[20,25]$.

\subsection{Proof of Theorem 3.2}

The first part is a direct consequence of Theorem 3.1 and the $L^{2}$ conservation law. It remains to prove the smoothing effect. Set

$$
R(t)=\int_{0}^{t} U\left(t-t^{\prime}\right) B\left(W\left(t^{\prime}\right)\right) W_{x}\left(t^{\prime}\right) \mathrm{d} t^{\prime}
$$

Due to a Sobolev embedding we obtain

$$
\|R(t)\|_{H^{s, 0} \times H^{s, 0}} \leq\|R\|_{X_{s} \times X_{s}} .
$$


Now a use of Propositions 3.1 and 3.2 yields

$$
\begin{aligned}
\|R(t)\|_{H^{s, 0} \times H^{s, 0}} & \leq c\left\{\left\|B\left(w_{1}, w_{2}\right)\right\|_{Y_{s}}+\left\|B\left(w_{1}, w_{2}\right)\right\|_{Y_{s}}+\left\|B\left(w_{1}, w_{2}\right)\right\|_{Y_{s}}\right\} \\
& \leq c\left\{\left\|w_{1}\right\|_{X}^{2}+\left\|w_{2}\right\|_{X}^{2}\right\} \\
& \leq \text { const. }
\end{aligned}
$$

This completes the proof of Theorem 3.2.

\section{Global Well-POSEDNESS IN $H^{s}\left(\mathbb{R}^{2}\right), s>0$}

Finally we shall show that the global well-posedness of (3) can be extended to initial data of higher smoothness. The difficulty is that there is no conservation of the $H^{s}\left(\mathbb{R}^{2}\right), s>0$ norms of the solutions. Nevertheless, as it is noticed in [8] one can overcome this difficulty by using the special form of the nonlinear estimate in the case of initial data of higher smoothness. We introduce the Fourier transform restriction norm

$$
\|u\|_{Z_{s}}^{2}=\int\langle\zeta\rangle^{2 s}\langle\sigma\rangle^{1+}\left(1+\frac{\langle\sigma\rangle^{\frac{7}{32}}}{\langle\xi\rangle^{\frac{1}{4}}}\right)^{2}|\widehat{u}(\tau, \zeta)|^{2} \mathrm{~d} \tau \mathrm{d} \zeta .
$$

Let $I \subset \mathbb{R}$ be an interval. Then we define the space $Z_{s}(I)$ equipped with the norm

$$
\|u\|_{Z_{s}(I)}=\inf _{w \in Z_{s}}\left\{\|w\|_{Z_{s}}, w(t)=u(t) \text { on } I\right\} .
$$

Similarly we define $X(I)$. Let $W(t)$ be a solution of (12) with $W(0) \in H^{s} \times H^{s}, s \geq 0$ (and hence in $L^{2}$ ) on the time interval $I=[0, \delta]$, where $\delta$ depends only on $\|W(0)\|_{L^{2}}$. Our aim is to show that $W \in Z_{s}([0, \delta])$. Using the elementary inequality

$$
\langle\zeta\rangle^{s} \leq c\left(\left\langle\zeta_{1}\right\rangle^{s}+\left\langle\zeta-\zeta_{1}\right\rangle^{s}\right)
$$

and the arguments of the proof of Proposition 3.2 and Section 3.4 of [25] one can obtain

$$
\begin{aligned}
\|W\|_{Z_{s}(I) \times Z_{s}(I)} \leq & c\|W(0)\|_{H^{s} \times H^{s}}+c \delta^{\theta}\left(\left\|w_{1}\right\|_{X(I)}\left\|w_{1}\right\|_{Z_{s}(I)}+\left\|w_{2}\right\|_{X(I)}\left\|w_{2}\right\|_{Z_{s}(I)}\right. \\
& \left.+\left\|w_{1}\right\|_{X(I)}\left\|w_{2}\right\|_{Z_{s}(I)}+\left\|w_{2}\right\|_{X(I)}\left\|w_{1}\right\|_{Z_{s}(I)}\right)
\end{aligned}
$$

where $\theta>0$. The local existence argument in $L^{2}$ provides the smallness of $\delta^{\theta}\left\|w_{1,2}\right\|_{X(I)}$ and hence one obtains a bound for $\left\|w_{1,2}\right\|_{Z_{s}(I)}$. The Sobolev inequality yields

$$
\sup _{t \in[0, \delta]}\left\|w_{1,2}(t)\right\|_{H^{s}} \leq c\left\|w_{1,2}\right\|_{Z_{s}([0, \delta])}
$$

Hence we control the $H^{s}$ norm of the solution on an interval of size depending only on the $L^{2}$ of the initial data. Thanks to the conservation of the $L^{2}$ norm we obtain a bound for the $H^{s}$ norm on any time interval. Therefore we have the following Theorem.

Theorem 4.1. Suppose that $a_{11} a_{22}-a_{12} a_{21}>0$. Then for any

$$
\left(u_{0}, v_{0}\right) \in H^{s}\left(\mathbb{R}^{2}\right) \times H^{s}\left(\mathbb{R}^{2}\right), \quad s \geq 0,
$$

such that

$$
|\xi|^{-1} \mathcal{F}\left(u_{0}\right)(\xi, \eta) \in \mathcal{S}^{\prime}\left(\mathbb{R}^{2}\right), \quad|\xi|^{-1} \mathcal{F}\left(v_{0}\right)(\xi, \eta) \in \mathcal{S}^{\prime}\left(\mathbb{R}^{2}\right)
$$


there exists a unique solution $(u, v)$ of the Cauchy problem (3). In addition the solution satisfy

$$
(u, v) \in C\left(\mathbb{R} ; H^{s}\left(\mathbb{R}^{2}\right)\right) \times C\left(\mathbb{R} ; H^{s}\left(\mathbb{R}^{2}\right)\right) .
$$

\section{Appendix A. A Remark on the CASE of the Gear-Grimshaw system}

Note that we have taken $r=0$ in (3) assuming that $c_{m}-c_{n}$ is very small comparatively to $\alpha$. If it is not the case then an additional difficulty appears because of the lower term perturbation of the system. In this appendix we shall show that one can overcome this difficulty in the absence of transverse effects. Thus consider the Gear-Grimshaw ( $c f .[12])$ system which we refer to in the introduction

$$
\left\{\begin{array}{c}
u_{t}+a_{11} u_{x x x}+a_{12} v_{x x x}+b_{1}(u v)_{x}+b_{2} u u_{x}+b_{3} v v_{x}=0 \\
v_{t}+a_{21} u_{x x x}+a_{22} v_{x x x}+r v_{x}+b_{4}(u v)_{x}+b_{5} u u_{x}+b_{6} v v_{x}=0 \\
u(0, x)=u_{0}(x), \quad v(0, x)=v_{0}(x) .
\end{array}\right.
$$

In both papers [3,4] $r$ is assumed to be zero, similarly to the present paper. For the approach of [4] the presence of $r$ does not affect the analysis, since the oscillatory integral estimates are still valid. On the other hand for the approach of [3] a technical difficulty appears when $r \neq 0$. Essentially one has to derive the bilinear estimates in Bourgain spaces with different phase functions. Our aim here is to show that one can get rid of this difficulty. We have the following Theorem.

Theorem 4.2. Assume that $r \neq 0$ and that the matrix $\left(a_{i j}\right)_{i, j \in\{1,2\}}$ has real distinct eigenvalues. Then the Cauchy problem (27) is globally well-posed for data $\left(u_{0}, v_{0}\right) \in L^{2}(\mathbb{R}) \times L^{2}(\mathbb{R})$.

Proof. Write (27) in the form

$$
\left\{\begin{array}{c}
U_{t}+A U_{x x x}+B U_{x}+C(U) U_{x}=0 \\
U(0)=U_{0}(x)
\end{array}\right.
$$

where $U=(u, v)^{t}$ and

$$
A=\left(\begin{array}{ll}
a_{11} & a_{12} \\
a_{21} & a_{22}
\end{array}\right), \quad B=\left(\begin{array}{cc}
0 & 0 \\
0 & r
\end{array}\right), \quad C=\left(\begin{array}{cc}
b_{2} u+b_{1} v & b_{1} u+b_{3} v \\
b_{5} u+b_{4} v & b_{4} u+b_{5} v
\end{array}\right) .
$$

Let $T \in \operatorname{GL}(2)$ such that $T^{-1} A T=\operatorname{diag}\left(\alpha_{+}, \alpha_{-}\right)$, where $\alpha_{ \pm}$are the eigenvalues of $A$. Let $U=T^{-1} V$. Then $V$ satisfies

$$
\left\{\begin{array}{c}
V_{t}+\operatorname{diag}\left(\alpha_{+}, \alpha_{-}\right) V_{x x x}+B_{1} V_{x}+C_{1}(V) V_{x}=0, \\
V(0)=T^{-1} U_{0},
\end{array}\right.
$$

where $B_{1}=T^{-1} B T$ and $C_{1}(V)=C\left(T^{-1} V\right) T^{-1}$. Note that $\operatorname{det} B_{1}=0$. Let $V(t, x)=\left(v_{1}(t, x), v_{2}(t, x)\right)^{t}$. Perform the scale change

$$
v_{1}(t, x)=w_{1}\left(t, \frac{x}{\alpha_{+}^{1 / 3}}\right), \quad v_{2}(t, x)=w_{2}\left(t, \frac{x}{\alpha_{-}^{1 / 3}}\right) .
$$

The equation satisfied by $W=\left(w_{1}, w_{2}\right)$ is

$$
W_{t}+W_{x x x}+B_{2} W_{x}+C_{2}(W) W_{x}=0,
$$


with some matrices $B_{2}$ and $C_{2}$. We now explicit the relationship between $B_{2}$ and $B_{1}$. Let

$$
B_{1}=\left(\begin{array}{ll}
b_{11} & b_{12} \\
b_{21} & b_{22}
\end{array}\right)
$$

Then

$$
B_{2}=\left(\begin{array}{cc}
\frac{1}{\alpha_{+}^{1 / 3}} b_{11} & \frac{1}{\alpha_{-}^{1 / 3}} b_{12} \\
\frac{1}{\alpha_{+}^{1 / 3}} b_{21} & \frac{1}{\alpha_{-}^{1 / 3}} b_{22}
\end{array}\right) .
$$

Hence $\operatorname{det} B_{2}=\operatorname{det} B_{1}=0$. Therefore $B_{2}$ has two real eigenvalues including zero. Moreover we can assume that there exists $T_{1} \in \mathrm{GL}(2)$ such that $T_{1}^{-1} B_{1} T_{1}=\operatorname{diag}(0, \gamma)$. Then $T^{-1} W:=g$ satisfies

$$
g_{t}+g_{x x x}+\operatorname{diag}(0, \gamma) g_{x}+C_{3}(g) g=0 .
$$

To keep the notations transparent let denote again $g=(u, v)^{t}$. Then (28) has the form

$$
\left\{\begin{array}{c}
u_{t}+u_{x x x}+\bar{c}_{1}(u v)_{x}+\bar{c}_{2} u u_{x}+\bar{c}_{3} v v_{x}=0 \\
v_{t}+v_{x x x}+\gamma v_{x}+\bar{c}_{4}(u v)_{x}+\bar{c}_{5} u u_{x}+\bar{c}_{6} v v_{x}=0 .
\end{array}\right.
$$

Next we define the functional spaces where the solutions of (29) belong to

$$
\begin{gathered}
X^{+}=\left\{u:\left\|\left\langle\tau-\xi^{3}\right\rangle^{\frac{1}{2}+} \widehat{u}(\tau, \xi)\right\|_{L^{2}}<\infty\right\}, \\
X^{-}=\left\{v:\left\|\left\langle\tau-\xi^{3}+\gamma \xi\right\rangle^{\frac{1}{2}+} \widehat{v}(\tau, \xi)\right\|_{L^{2}}<\infty\right\} .
\end{gathered}
$$

Now we state the bilinear estimates needed for the proof of Theorem 5.1.

Proposition 4.1. The following estimates hold

$$
\begin{aligned}
\left\|\partial_{x}(u v)\right\|_{X^{+}} & \leq\|u\|_{X^{+}}\|v\|_{X^{+}} \\
\left\|\partial_{x}(u v)\right\|_{X^{+}} & \leq\|u\|_{X^{+}}\|v\|_{X^{-}} \\
\left\|\partial_{x}(u v)\right\|_{X^{+}} & \leq\|u\|_{X^{-}}\|v\|_{X^{-}} \\
\left\|\partial_{x}(u v)\right\|_{X^{-}} & \leq\|u\|_{X^{+}}\|v\|_{X^{+}} \\
\left\|\partial_{x}(u v)\right\|_{X^{-}} & \leq\|u\|_{X^{+}}\|v\|_{X^{-}} \\
\left\|\partial_{x}(u v)\right\|_{X^{-}} & \leq\|u\|_{X^{-}}\|v\|_{X^{-}}
\end{aligned}
$$

Obviously the linear estimates in the proof of Theorem 5.1 are similar to these of the proof of Theorem 3.1. Then a standard fixed point argument completes the proof. Hence it remains to prove Proposition 5.1.

Proof of Proposition 5.1. We shall prove (31). The proofs of the other estimates are similar (some of them are simpler). Set

and furthermore

$$
\sigma=\tau-\xi^{3}, \quad \sigma_{1}=\tau_{1}-\xi_{1}^{3}, \quad \sigma_{2}=\tau-\tau_{1}-\left(\xi-\xi_{1}\right)^{3}+\gamma\left(\xi-\xi_{1}\right)
$$

$$
J=\iint \frac{|\xi| \widehat{u}\left(\tau_{1}, \xi_{1}\right) \widehat{v}\left(\tau-\tau_{1}, \xi-\xi_{1}\right) \widehat{w}(\tau, \xi)}{\langle\sigma\rangle^{\frac{1}{2}-}\left\langle\sigma_{1}\right\rangle^{\frac{1}{2}+}\left\langle\sigma_{2}\right\rangle^{\frac{1}{2}+}} \mathrm{d} \tau \mathrm{d} \xi \mathrm{d} \tau_{1} \mathrm{~d} \xi_{1}
$$


We want to obtain a bound

$$
J \leq c\|u\|_{L^{2}}\|v\|_{L^{2}}\|w\|_{L^{2}} .
$$

Assume first $|\xi| \leq c_{0}$ ( $c_{0}$ to be fixed later). Then we have the next version of Strichartz inequality

$$
\left\|\mathcal{F}^{-1}\left(\left\langle\tau-\xi^{3}+\alpha \xi\right\rangle^{-b}|\widehat{u}(\tau, \xi)|\right)\right\|_{L^{4}} \leq\|u\|_{L^{2}},
$$

for $b>1 / 3$ and $\alpha \in \mathbb{R}$. Actually in [20] the estimate (36) is proved with $\alpha=0$. However the lower order perturbation of the symbol does not affect the analysis since the essential assumption to prove (36) lies on the second derivative of the phase function. Now a use of (36), Hölder inequality and Plancherel identity gives a bound for the contribution to $J$ of the region where $|\xi|$ is bounded. Let now $|\xi|$ be away of zero. Denote by $\tilde{J}$ the contribution of this region to $J$. Cauchy-Schwarz inequality yields

$$
\tilde{J} \leq \int I(\tau, \xi)\left(\int\left|\widehat{u}\left(\tau_{1}, \xi_{1}\right) \widehat{v}\left(\tau-\tau_{1}, \xi-\xi_{1}\right)\right|^{2} \mathrm{~d} \tau_{1} \mathrm{~d} \xi_{1}\right)^{\frac{1}{2}} \widehat{w}(\tau, \xi) \mathrm{d} \tau \mathrm{d} \xi,
$$

where

A use of (20) yields

$$
I(\tau, \xi)=\frac{c|\xi|}{\langle\sigma\rangle^{\frac{1}{2}-}}\left(\iint \frac{\mathrm{d} \tau_{1} \mathrm{~d} \xi_{1}}{\left\langle\sigma_{1}\right\rangle^{\frac{1}{2}+}\left\langle\sigma_{2}\right\rangle^{\frac{1}{2}+}}\right)^{\frac{1}{2}}
$$

Perform a change of variables

$$
I(\tau, \xi) \leq \frac{c|\xi|}{\langle\sigma\rangle^{\frac{1}{2}-}}\left(\int \frac{\mathrm{d} \xi_{1}}{\left\langle\sigma_{1}+\sigma_{2}\right\rangle^{\frac{1}{2}+}}\right)^{\frac{1}{2}} .
$$

Then a straightforward computation leads to

$$
\mu=\sigma_{1}+\sigma_{2}
$$

$$
\mathrm{d} \xi_{1}=\frac{c \mathrm{~d} \mu}{|\xi|^{\frac{1}{2}}\left|\mu-\sigma-\gamma \xi-\frac{\left(3 \xi^{2}-\gamma\right)^{2}}{12 \xi}\right|^{\frac{1}{2}}} .
$$

Hence a use of (21) yields

We claim that

$$
I(\tau, \xi) \leq \frac{c|\xi|^{\frac{3}{4}}}{\langle\sigma\rangle^{\frac{1}{2}-}\left\langle\sigma+\gamma \xi+\frac{\left(3 \xi^{2}-\gamma\right)^{2}}{12 \xi}\right\rangle^{\frac{1}{4}}} .
$$

$$
\langle\sigma\rangle^{\frac{1}{2}-}\left\langle\sigma+\gamma \xi+\frac{\left(3 \xi^{2}-\gamma\right)^{2}}{12 \xi}\right\rangle^{\frac{1}{4}} \geq c|\xi|^{\frac{3}{4}} .
$$

Actually we have

$$
|\sigma|+\left|\sigma+\gamma \xi+\frac{\left(3 \xi^{2}-\gamma\right)^{2}}{12 \xi}\right|^{\frac{1}{4}} \geq \frac{3}{4}|\xi|^{3}-\frac{|\gamma|}{2}|\xi|-\frac{\gamma^{2}}{12} \frac{1}{|\xi|} \geq \frac{3}{8}|\xi|^{3},
$$

for $|\xi|$ sufficiently large. Hence we proved $(37)$ and therefore $I(\tau, \xi)$ is bounded. Another use of Cauchy-Schwarz inequality completes the proof of (31).

Remark 3. With the arguments of this Appendix one can prove local well-posedness with data in $L^{2}$ for the equation

where $p(D)$ is a real Fourier multiplier with symbol

$$
u_{t}+i p(D) u+u u_{x}=0,
$$

$$
p(\xi)=\xi^{3}+\text { lower order terms }
$$


for $\xi \gg 1$. In particular when $p(\xi)=\xi^{3}+\xi|\xi|$ one obtains that the Benjamin equation is well-posed in $L^{2}$, which is the result of [19].

\section{Appendix B. Proof of Proposition 2.1}

We shall use the following Sobolev inequality which holds for any $s_{1}>1 / 2, \quad s_{2}>1 / 2$

$$
\|u\|_{L^{\infty}} \leq c\|u\|_{H^{s_{1}, s_{2}}} .
$$

The inequality (38) is an anisotropic version of the classical Sobolev inequality. In order to prove it we may use the inverse Fourier transform formula, the Cauchy-Schwarz inequality and that

$$
\left\langle\xi_{1}\right\rangle^{-s_{1}}\left\langle\xi_{2}\right\rangle^{-s_{2}} \in L_{\xi_{1}, \xi_{2}}^{2}, \text { for } s_{1}>1 / 2, s_{2}>1 / 2
$$

In order to prove Proposition 2.1 we shall use the arguments of Coifman and Meyer [10] (cf. also [2,5,9]). The statement of Proposition 2.1 is an anisotropic version of the fact that the classical Sobolev spaces $H^{s}\left(\mathbb{R}^{n}\right)$ is an algebra for $s>n / 2$. Our proof relies on an anisotropic Littlewood-Paley decomposition of functions defined on $\mathbb{R}^{2}$. We refer to $[11,16]$, where anisotropic Littlewood-Paley decompositions are used in other contexts. Let $\psi \in C_{0}^{\infty}(\mathbb{R}), \psi(\xi)=1$ for $|\xi| \leq 1 / 2$ and $\psi(\xi)=0$ for $|\xi| \geq 1$. Let $\phi(\xi)=\psi(\xi / 2)-\psi(\xi)$. Then clearly

$$
1=\psi(\xi)+\sum_{p=0}^{\infty} \phi\left(2^{-p} \xi\right)
$$

Now we define the Fourier multiplier operators $\Delta_{p}^{x}, \Delta_{p}^{y}, S_{p}^{x}, S_{p}^{y}, S_{p q}$ as follows

$$
\begin{aligned}
& \Delta_{0}^{x}=\psi\left(D_{x}\right), \quad \Delta_{0}^{y}=\psi\left(D_{y}\right), \quad \Delta_{p}^{x}=\phi\left(2^{-p+1} D_{x}\right), \quad \Delta_{q}^{y}=\phi\left(2^{-q+1} D_{y}\right) \\
& S_{p}^{x}=\sum_{q \leq p} \Delta_{p}^{x}, \quad S_{p}^{y}=\sum_{q \leq p} \Delta_{p}^{y}, \quad S_{p q}=\sum_{p_{1} \leq p} \sum_{q_{1} \leq q} \Delta_{p_{1}}^{x} \Delta_{q_{1}}^{y}, \quad p, q=1,2, \ldots
\end{aligned}
$$

Then we have the following Littlewood-Paley type decomposition of $u \in \mathcal{S}^{\prime}\left(\mathbb{R}^{2}\right)$

$$
u=\sum_{p \geq 0} \sum_{q \geq 0} \Delta_{p q} u
$$

where $\Delta_{p q}=\Delta_{p}^{x} \Delta_{q}^{y}$. Let $u, v \in H^{s_{1}, s_{2}}\left(\mathbb{R}^{2}\right)$. Then we may represent the product $u v$ as

$$
u v=I_{1}(u, v)+I_{2}(u, v)+I_{3}(u, v)+I_{4}(u, v)
$$


where

$$
\begin{aligned}
& I_{1}(u, v)=\sum_{p_{2}, q_{2}} S_{p_{2} q_{2}} u \cdot \Delta_{p_{2} q_{2}} v:=\sum_{p_{2}, q_{2}} I_{1}^{p_{2} q_{2}}(u, v), \\
& I_{2}(u, v)=\sum_{p_{2}, q_{1}} S_{p_{2}}^{x} \Delta_{q_{1}}^{y} u \cdot \Delta_{p_{2}}^{x} S_{q_{1}-1}^{y} v:=\sum_{p_{2}, q_{1}} I_{2}^{p_{2} q_{1}}(u, v), \\
& I_{3}(u, v)=\sum_{p_{1}, q_{2}} \Delta_{p_{1}}^{x} S_{q_{2}}^{y} u \cdot S_{p_{1}-1}^{x} \Delta_{q_{2}}^{y} v:=\sum_{p_{1}, q_{2}} I_{3}^{p_{1} q_{2}}(u, v), \\
& I_{4}(u, v)=\sum_{p_{1}, q_{1}} \Delta_{p_{1} q_{1}} u \cdot S_{\left(p_{1}-1\right)\left(q_{1}-1\right)} v:=\sum_{p_{1}, q_{1}} I_{4}^{p_{1} q_{1}}(u, v) .
\end{aligned}
$$

A scaling argument easily yields that the operators $S_{p}^{x}, S_{p}^{y}, S_{p q}$ are bounded in $L^{\infty}\left(\mathbb{R}^{2}\right)$ with an operator norm independent of $p, q$ ( $c f$. [2]. Lemma 1.1.2 for example). Now we use the Hölder inequality and a one dimensional Sobolev inequality in order to bound the $L^{2}\left(\mathbb{R}^{2}\right)$ norm of $I_{2}^{p_{2} q_{1}}(u, v)$

$$
\begin{aligned}
\left\|I_{2}^{p_{2} q_{1}}(u, v)\right\|_{L^{2}} & \leq\left\|S_{p_{2}}^{x} \Delta_{q_{1}}^{y} u\right\|_{L_{x}^{\infty} L_{y}^{2}}\left\|\Delta_{p_{2}}^{x} S_{q_{1}-1}^{y} v\right\|_{L_{x}^{2} L_{y}^{\infty}} \\
& \leq c_{p_{2} q_{1}} \cdot 2^{-q_{1} s_{2}}\|u\|_{H^{s_{1}, s_{2}}} \cdot 2^{-p_{2} s_{1}}\|v\|_{H^{s_{1}, s_{2}}},
\end{aligned}
$$

where $c_{p_{2} q_{1}} \in l_{p_{2} q_{1}}^{2}$. We have the following support property of $I_{2}^{p_{2} q_{1}}(u, v)$

$$
\operatorname{supp}\left(\mathcal{F}\left(I_{2}^{p_{2} q_{1}}(u, v)\right)\right) \subset\left\{\left(\xi_{1}, \xi_{2}\right):\left|\xi_{1}\right| \leq c \cdot 2^{p_{2}},\left|\xi_{2}\right| \leq c \cdot 2^{q_{1}}\right\} .
$$

Hence summing over $p_{2}$ and $q_{1}$ in (39) yields

$$
\left\|I_{2}(u, v)\right\|_{H^{s_{1}, s_{2}}} \leq c\|u\|_{H^{s_{1}, s_{2}}}\|v\|_{H^{s_{1}, s_{2}}} .
$$

Now we shall estimate $\left\|I_{1}(u, v)\right\|_{H^{s_{1}, s_{2}}}$. Hölder inequality and (38) yield

$$
\begin{aligned}
\left\|I_{1}^{p_{2} q_{2}}(u, v)\right\|_{L^{2}} & \leq\left\|S_{p_{2} q_{2}} u\right\|_{L_{x, y}^{\infty}}\left\|\Delta_{p_{2} q_{2}} v\right\|_{L_{x, y}^{2}} \\
& \leq c_{p_{2} q_{2}} \cdot 2^{-p_{2} s_{1}} \cdot 2^{-q_{2} s_{2}}\|u\|_{H^{s_{1}, s_{2}}}\|v\|_{H^{s_{1}, s_{2}}},
\end{aligned}
$$

where $c_{p_{2} q_{2}} \in l_{p_{2} q_{2}}^{2}$. Now summing over $p_{2}, q_{2}$ we obtain

$$
\left\|I_{1}(u, v)\right\|_{H^{s_{1}, s_{2}}} \leq\|u\|_{H^{s_{1}, s_{2}}}\|v\|_{H^{s_{1}, s_{2}}}
$$

similarly to the estimate for $\left\|I_{2}(u, v)\right\|_{H^{s_{1}, s_{2}}}$. The estimate of $\left\|I_{4}(u, v)\right\|_{H^{s_{1}, s_{2}}}$ is similar to that of $\left\|I_{1}(u, v)\right\|_{H^{s_{1}, s_{2}}}$ and the bound for $\left\|I_{3}(u, v)\right\|_{H^{s_{1}, s_{2}}}$ is similar to that of $\left\|I_{2}(u, v)\right\|_{H^{s_{1}, s_{2}}}$. Hence we obtain that

$$
\|u v\|_{H^{s_{1}, s_{2}}} \leq\|u\|_{H^{s_{1}, s_{2}}}\|v\|_{H^{s_{1}, s_{2}}}
$$

for $s_{1}>1 / 2, s_{2}>1 / 2$ which completes the proof of Proposition 2.1. 
Remark 4. Arguments similar to that presented above allows us to establish the following product estimate in the anisotropic Sobolev spaces $H^{s_{1}, s_{2}}\left(\mathbb{R}^{2}\right)$ for $s_{1}$ and $s_{2}$ positive and $u, v \in H^{s_{1}, s_{2}}\left(\mathbb{R}^{2}\right) \cap L^{\infty}\left(\mathbb{R}^{2}\right) \cap H_{x}^{s_{1}} L_{y}^{\infty}\left(\mathbb{R}^{2}\right) \cap$ $L_{x}^{\infty} H_{y}^{s_{2}}\left(\mathbb{R}^{2}\right)$

$$
\|u v\|_{H^{s_{1}, s_{2}}} \leq c\left(\|u\|_{H^{s_{1}, s_{2}}}\|v\|_{L^{\infty}}+\|u\|_{H_{x}^{s_{1}} L_{y}^{\infty}}\|v\|_{L_{x}^{\infty} H_{y}^{s_{2}}}+\|u\|_{L_{x}^{\infty} H_{y}^{s_{2}}}\|v\|_{H_{x}^{s_{1}} L_{y}^{\infty}}+\|u\|_{L^{\infty}}\|v\|_{H^{s_{1}, s_{2}}}\right) .
$$

\section{REFERENCES}

[1] J. Albert, J. Bona and J.C. Saut, Model equations for waves in stratified fluids. Proc. Roy. Soc. Lond. A 453 (1997) 1213-1260.

[2] S. Alinhac and P. Gérard, Opérateurs pseudo-différentiel et théorème de Nash-Moser. Éditions du CNRS, EDP Sciences (1991).

[3] J.M. Ash, J. Cohen and G. Wang, On strongly interacting internal solitary waves. J. Fourier Anal. and Appl. 5 (1996) $507-517$.

[4] J. Bona, G. Ponce, J.C. Saut and M. Tom, A model system for strong interaction between internal solitary waves. Comm. Math. Phys. 143 (1992) 287-313.

[5] J.-M. Bony, Calcul symbolique et propagation des singularités pour les équations aux dérivées partielles non linéaires. Annales de l'ENS 14 (1981) 209-246.

[6] J. Bourgain, Fourier transform restriction phenomena for certain lattice subsets and application to nonlinear evolution equations I. Schrödinger equations. GAFA 3 (1993) 107-156.

[7] J. Bourgain, Fourier transform restriction phenomena for certain lattice subsets and application to nonlinear evolution equations II. The KdV equation. GAFA 3 (1993) 209-262.

[8] J. Bourgain, On the Cauchy problem for the Kadomtsev-Petviashvili equation. GAFA 3 (1993) 315-341.

[9] J.-Y. Chemin, Fluid parfaits incompressibles. Astérisque 230 (1995).

[10] R. Coifman and Y. Meyer, Au delà des operateurs pseudodifférentiels. Astérisque $\mathbf{5 7}$ (1978).

[11] I. Gallagher, Applications of Schochet's methods to parabolic equations. J. Math. Pures Appl. 77 (1998) 989-1054.

[12] J.A. Gear and R. Grimshaw, Weak and strong interactions between internal solitary waves. Stud. Appl. Math. 65 (1984) 235-258.

[13] J. Ginibre, Le problème de Cauchy pour des EDP semi-linéaires périodiques en variables d'espace (d'après Bourgain). Séminaire Bourbaki 796, Astérique 237 (1995) 163-187.

[14] J. Ginibre, Y. Tsutsumi and G. Velo, On the Cauchy problem for the Zakharov system. J. Funct. Anal. 151 (1997) $384-436$.

[15] R. Grimshaw, Y. Zhu, Oblique interactions between internal solitary waves. Stud. Appl. Math. 92 (1994) 249-270.

[16] D. Iftimie, The resolution of the Navier-Stokes equations in anisotropic spaces. Revista Matematica Ibero-Americana 15 (1999) $1-36$.

[17] R.J. Iório Jr, W.V.L. Nunes, On equations of KP-type. Proc. Roy. Soc. Edinburgh A 128 (1998) 725-743.

[18] P. Isaza, J. Mejia and V. Stallbohm, El problema de Cauchy para la ecuacion de Kadomtsev-Petviashvili (KP-II) en espacios de Sobolev $H^{s}, s>0$, preprint (1997).

[19] F. Linares, $L^{2}$ global well-posedness of the initial value problem associated to the Benjamin equation. J. Differential Equations 152 (1999) 377-393.

[20] C. Kenig, G. Ponce and L. Vega, A bilinear estimate with applications to the KdV equations. J. AMS 9 (1996) 573-603.

[21] C. Kenig, G. Ponce and L. Vega, Quadratic forms for $1-D$ semilinear Schrödinger equation. Trans. Amer. Math. Soc. 348 (1996) 3323-3353.

[22] J.C. Saut, Remarks on the generalized Kadomtsev-Petviashvili equations. Indiana Univ. Math. J. 42 (1993) $1017-1029$.

[23] R. Strichartz, Restriction of Fourier transforms to quadratic surfaces and decay of solutions of wave equations. Duke Math. J. 44 (1977) 705-714.

[24] H. Takaoka, Well-posedness for the Kadomtsev-Petviashvili II equation, preprint (1998).

[25] N. Tzvetkov, Global low regularity solutions for Kadomtsev-Petviashvili equation. Diff. Int. Eq. (to appear). 